\title{
FORMATION AND EVOLUTION OF X-RAY CLUSTERS: A HYDRODYNAMIC SIMULATION OF THE INTRACLUSTER MEDIUM
}

\author{
AUgust E. EVRaRD \\ Department of Astronomy, University of California, Berkeley \\ Received 1989 June 22; accepted 1990 May 2
}

\begin{abstract}
The X-ray-emitting intracluster medium (ICM) in clusters of galaxies is studied using a combined threedimensional hydrodynamic and $N$-body simulation algorithn with the assumption that clusters formed via hierarchical clustering in a cold dark matter-dominated universe with ICM fraction $\Omega_{\mathrm{ICM}}=0.1$. The ICM is treated as an ideal $\gamma=5 / 3$ gas undergoing shock heating within the self-consistency evolving dark matter potential.

The evolution of a single, Coma-richness cluster is examined in detail. A core of $\sim 10^{13} M_{\odot}$ of gas collapses by $z=1$ and grows through mergers and accretion of surrounding material. The process can be crudely defined by a shock front moving outward at $\sim 400 \mathrm{~km} \mathrm{~s}^{-1}$ reaching a radius $\sim 5 h_{50}^{-1} \mathrm{Mpc}$ at the present epoch. The $2 \times 10^{14} h_{50}^{-1} M_{\odot}$ of gas within an Abell radius at $z=0$ is close to isothermal at $T=7.2 \mathrm{keV}$ with evidence for a modest temperature inversion in the cluster center. Polytropic models provide a poor description of the ICM thermodynamic state. The density profile of the cluster shows no resolved core radius, although a flattening of the logarithmic slope is apparent at radii $r \lesssim 500 h_{50}^{-1} \mathrm{kpc}$. The total $2-10 \mathrm{keV}$ luminosity of $7.5 \times 10^{44} h_{50}$ ergs $\mathrm{s}^{-1}$ is therefore subject to an uncertain core contribution. A Sunyaev-Zel'dovich central decrement of roughly $0.5 \mathrm{mK}$ would be expected from this cluster at redshifts $z \lesssim 0.25$ at $1^{\prime}$ resolution. The signal drops below $0.1 \mathrm{mK}$ at $z=0.5$

Investigation of the standard hydrostatic isothermal $\beta$-model shows that estimates of the specific energy ratio $\beta \equiv \sigma^{2} /\left(k T / \mu m_{p}\right)$ based on surface brightness profiles are systematically biased. The discrepancy arises from the existence of residual kinetic energy in the shock-heated gas and poor modeling of the underlying binding mass distribution. Simple binding mass estimates based on this model underestimate the actual binding mass within an Abell radius by $\sim 30 \%$. The mass contained within an Abell radius for Coma and A2256 is estimated to be 2.4 and $2.6 \times 10^{15} h_{50}^{-1} M_{\odot}$, respectively, after correcting for these effects.
\end{abstract}

Subject headings: galaxies: clustering - galaxies: intergalactic medium — galaxies: X-rays — hydrodynamics

\section{INTRODUCTION}

Rich clusters of galaxies are observed to be strong sources of diffuse X-ray emission produced by optically thin thermal bremsstrahlung from a hot $\left(T \sim 10^{8} \mathrm{~K}\right)$ intracluster plasma (see reviews by Forman and Jones 1982, Sarazin 1986, Fabian 1988, and Mushotzky 1988). Since the gas represents at least part of the baryonic component of the universe which does not now reside in galaxies, understanding the formation and evolution of the hot gas would provide valuable information on the formation of galaxies and large-scale structure in general. At present, there remain several unresolved theoretical issues regarding the history of the gas comprising the intracluster medium (ICM) including the following:

1. Origin of the gas.-Is the ICM simply primordial material left over from galaxy formation which is enriched by ram pressure stripping of infalling galaxies (Gunn and Gott 1972), or was the ICM produced entirely by ejection of gas from galaxies after cluster formation (Yahil and Ostriker 1973; Larson and Dinerstein 1975; Cowie and Perrenod 1978)?

2. Thermal history.-How do the gas temperature and density evolve with time? Is shock heating through gravitational collapse sufficient to produce the emission observed in $\mathrm{X}$-ray clusters? Is the ICM in hydrostatic equilibrium within the cluster potential?

3. Temperature profile.-Is the gas temperature profile $T(r)$ nearly isothermal? Do polytropic models provide a better description?
4. Relation to dark matter.-Does the gas distribution follow that of the underlying dark matter? How well do present models estimate the binding mass of clusters?

This paper (and a following paper, Evrard 1990b, hereafter Paper II) presents numerical simulations designed to provide some answers to the above questions within the context of hierarchical clustering in a cold dark matter (CDM) dominated universe (Peebles 1982; Blumenthal et al. 1984; Davis et al. 1985). The universe is assumed to have density equal to the closure value $\Omega=1$, with a mass fraction of baryons in the ICM $\Omega_{\mathrm{ICM}}=0.1$ and a Hubble constant $H_{0}=50 h_{50} \mathrm{~km} \mathrm{~s}^{-1}$ $\mathrm{Mpc}^{-1}$. In this cosmogony, rich clusters form from gravitational collapse of suitably filtered overdense peaks in the initial density field viewed at some early, linear epoch (Bardeen et al. 1986). Scaling relations for the expected temperature, density, and luminosity of clusters depend on the height of the peak and the chosen mass scale (Kaiser 1986). In this way, a range of cluster richness can be accommodated by varying the height of the initial peak density at a mass scale relevant to rich clusters.

The numerical models approximate X-ray clusters as twocomponent systems consisting of collisionless dark matter and a collisional gas of baryons representing the ICM. The ICM is assumed to be a fully ionized plasma of primordial composition and is treated as an ideal gas with ratio of specific heats $\gamma=5 / 3$. Shock heating is included, but radiative cooling is ignored. These simulations lack sufficient dynamic range to accurately resolve cooling flows within clusters or to resolve 
the formation and evolution of galaxies within the cluster. The question of the origin of the ICM will therefore not be addressed by this work. Instead, I will assume that galaxies in clusters have little effect on the thermal history of the bulk of the ICM. In the CDM model, this may arise "naturally" due to the inefficiency of galaxy formation required for biasing galaxies relative to the total mass of the universe (Bardeen et al. 1986; Frenk et al. 1988). With this point of view, there are two ways to explain the roughly half-solar ICM iron abundance (Mushotzky 1984)-preenrichment of the gas during an epoch of galaxy formation preceeding cluster collapse and/or passive enrichment resulting from stripping of enriched galactic gas as galaxies infall into the highly pressurized ICM (Gunn and Gott 1972; Takeda, Nulsen, and Fabian 1984).

Producing a simulated cluster is a two-stage process. First, a random realization of linear protocluster fields is generated using the path integral technique developed by Bertschinger (1987). There linear fields are then propagated into the nonlinear regime using the combined $N$-body/three-dimensional hydrodynamical code P3MSPH (Evrard 1988).

These models represent the first treatment of the hydrodynamic evolution of the ICM in a fully self-consistent, threedimensional, time-varying potential. Early one-dimensional simulations by Gull and Northover (1975), Lea (1976), and Cowie and Perrenod (1978) assumed spherical symmetry and a fixed gravitational potential into which the gas fell, essentially from infinity. These simulations confirmed that thermal bremsstrahlung, from gas shock-heated within characteristic cluster potential wells, could produce roughly the observed level of X-ray emission. Perrenod (1978) added a time-varying spherical potential adapted from White's (1976) $N$-body model of the Coma cluster. The secular deepening of the cluster potential led to a slowly increasing X-ray luminosity and temperature. Cavaliere et al. (1986) embedded gas within a collisionless $N$-body model by assuming hydrostatic equilibrium held at all times throughout the cluster. The emission morphologies exhibited by their model appeared similar to those observed by Einstein (Forman and Jones 1982).

The approach taken here represents an improvement over previous work in that the hydrodynamics is calculated selfconsistently in the evolving potential well of collisionless dark matter. Also, the initial conditions for the protocluster density and velocity fields are not ad hoc but rather are random realizations of the initial fields expected in a CDM-dominated universe. This allows specific statements to be made regarding expectations within the context of the CDM model.

In principle, knowledge of the equilibrium plasma distribution can be used to gain insight into the structure of the underlying potential and hence the distribution of binding mass within the cluster. Such mass estimates have broad cosmological implications on the amount of dark matter in the universe. In practice, uncertainties (primarily from lack of spatially resolved cluster temperatures) make estimating binding masses of clusters from X-ray data highly modeldependent (Cowie, Henriksen, and Mushotzky 1987; Fabricant, Kent, and Kurtz 1989; Hughes 1989). Numerical simulations provide a useful forum for investigating any systematic effects in current mass estimation techniques.

A related issue is the " $\beta$-discrepancy" of X-ray clusters. The quantity $\beta \equiv \sigma^{2} /\left(k T / \mu m_{p}\right)$, where $\sigma$ is the one-dimensional velocity dispersion of galaxies in the cluster, $T$ is the gas temperature, $\mu$ is the mean molecular weight, and $m_{p}$ is the proton mass, is a measure of the ratio of specific kinetic energy in galaxies to the specific thermal energy of particles in the plasma. The hydrostatic isothermal $\beta$-model (Cavaliere and Fusco-Fermiano 1976; Bahcall and Sarazin 1977; Jones and Forman 1984) has been widely used to describe the mass profiles of clusters. In this model, the density profile of the gas follows the form

$$
\rho_{g}(r)=\rho_{g, 0}\left[1+\left(r / r_{c}\right)^{2}\right]^{-3 \beta / 2},
$$

where $r_{c}$ is the cluster core radius, $\rho_{g, 0}$ is the central gas density, and $\beta$ is the ratio of specific energies defined above. Since the gas is assumed isothermal, the observed X-ray surface brightness scales with projected separation $\theta$ as

$$
\Sigma_{x}(\theta) \propto \theta^{-6 \beta+1}
$$

for radii outside the core region. With this model, an estimate of the ratio of specific energies can be made simply by fitting the outer surface brightness profile and estimating a value $\beta_{\mathrm{fit}}$. Values of $\beta_{\mathrm{fit}} \sim 0.6-0.8$ are typically found this way (Jones and Forman 1984) with the ensuring interpretation that the ICM is hotter and more extended than the galaxies.

However, these estimates of $\beta$ are not born out by direct spectroscopic measurements of the gas temperature and optically determined galaxy velocity dispersion which typically produce values $\beta_{\text {spec }} \simeq 1.2$ (Mushotzky 1984; Sarazin 1986; Fabian 1988; Mushotzky 1988), although uncertainties of $\gtrsim 20 \%$ are common. The interpretation of these values is the opposite - the gass is cooler and less extended than the galaxies. The discrepancy between the numerical values of $\beta_{\text {spec }}$ and $\beta_{\mathrm{fit}}$ has generated a considerable amount of confusion because of the opposing interpretations they represent. Interestingly, the simulated cluster exhibits the same " $\beta$ discrepancy" allowing the root cause of this problem to be examined in detail.

Recently, Edge (1990) has provided evidence from EXOSAT cluster observations that values of $\beta_{\mathrm{fit}}$ and $\beta_{\text {spec }}$ are both less than unity in low-temperature, low-luminosity clusters. However, the classic discrepancy $\beta_{\text {spec }}>1>\beta_{\text {fit }}$ persists in the richest clusters of his sample. The implications of these observations are not yet understood (David, Forman, and Jones 1990; Edge 1990; Evrard 1990a; White 1990).

The outline of the paper is as follows. Section II provides some details on the numerical procedure. The cluster evolution is followed in $\S$ III and issues regarding $\beta$ and mass estimation are examined. Section IV provides a brief comparison of the simulated cluster with Coma and A2256. The following section provides tests of the sensitivity of the results of the numerical parameters in the model. A final section provides a summary and discussion.

\section{METHOD}

\section{a) Cosmological Framework}

In hierarchical clustering models, rich clusters form at peaks in the density field smoothed on a mass scale appropriate for rich clusters $M \sim 10^{15} M_{\odot}$ (Kaiser 1986). For the CDM model in particular, the postrecombination density field is imprinted with linear fluctuations described as a random phase superposition of waves with amplitudes governed by a power spectrum $P(k)$ calculated as a transfer function applied to a primordial, adiabatic, constant curvature spectrum of fluctuations (Bond and Efstathiou 1984). Within a sphere of radius $x$ which, on average, contains a mass $M$, this leads to a Gaussian distribution of initial overdensities $\delta=\rho(x) /\langle\rho(x)\rangle-1$ with 
variance

$$
\sigma^{2}(M)=\frac{1}{(2 \pi)^{3}} \int d^{3} k P(k) W_{M}^{2}(k),
$$

where $W_{M}(k)=3(k x)^{-3}[\sin (k x)-k x \cos (k x)]$ is a window function appropriate to smoothing fluctuations within the sphere containing mass $M$. The initial fluctuations are therefore normally distributed in the variable

$$
v \equiv \delta / \sigma(M) \text {. }
$$

The theory of the statistics of peaks in Gaussian random fields developed by Bardeen et al. (1986) provides $n_{\mathrm{pk}}(v)$, the number density of peaks with height greater than $v$ for the density field smoothed with a Gaussian filter $W_{M}(k)=\exp \left(-k^{2} R_{f}^{2} / 2\right)$. With $R_{f}=10 h_{50}^{-1} \mathrm{Mpc}$ (suitable for filtering on a mass scale $M_{f}=10^{15} h_{50}^{-1} M_{\odot}$ ), the observed number density of $R \geq 1$ Abell clusters $n_{\mathrm{A}} \simeq 6 \times 10^{-7} h_{50}^{3} \mathrm{Mpc}^{-3}$ is reached for peaks above a threshold value $v_{t}=2.7$. This value of $v_{t}$ is consistent with the idea that $\sim 1 \%$ of the mass in the universe is in rich clusters (Kaiser 1986), since the fraction of perturbations satisfying the threshold condition is given by the complimentary error function erfc $\left(v_{t}\right)=0.007$.

In this paper, we concentrate on the details of the evolution of a single cluster arising from a peak of height $v=4.3$. The CDM spectrum normalization is fixed such that the present rms, linearly extrapolated mass fluctuations in a sphere of radius $16 h_{50}^{-1} \mathrm{Mpc}$ is 0.6 . The equivalent "bias parameter," defined as $b \equiv 1 / \sigma\left(16 h_{50}^{-1} \mathrm{Mpc}\right)$, has a value $b=1.7$. This normalization is intermediate between the values $b=2.5$ advocated by the numerical experiments of Davis et al. (1985) and the value $b \simeq 1.5$ inferred from observations of large-scale velocity fields (Kaiser and Lahav 1989; Bertschinger and Juszkiewicz 1988; Górski et al. 1989). A simple model for the abundance of rich clusters as a function of velocity dispersion indicates that a value $b=1.7$ can reproduce the observed abundances of nearby rich clusters (Evrard 1989). Given the protocluster peak height $v=4.3$, the expected abundance of peaks of this height or higher is $n=9 \times 10^{-9} h_{50}^{3} \mathrm{Mpc}^{-3}$ (Bardeen et al. 1986), implying that a cluster as rich as the one simulated here should be found within a redshift $z \sim 0.05$.

\section{b) Generating Initial Conditions}

A technique for sampling random fields under constraints developed by Bertschinger (1987) is used to generate initial conditions. The "path integral" method produces a mean field which satisfies a set of linear input constraints, to which is added a random field orthogonal to the constraints drawn from a suitably modified power spectrum. The method constructs the density field on a cubic lattice of side $N$ representing a periodic cube of length $L$. The imposed constraints can take any (nonsingular) form; here we use a simple constraint that the density field convolved with a Gaussian window of size $R_{f}=10 h_{50}^{-1} \mathrm{Mpc}$ equal an imposed value $v_{c} \sigma\left(M_{f}\right)$ at the center of the box. That is

$$
\begin{aligned}
\sigma\left(\boldsymbol{r}_{c}\right) & =\left(\frac{L}{N}\right)^{3}\left[\frac{1}{(2 \pi)^{3 / 2} R_{f}^{3}}\right] \sum_{k} \frac{\delta \rho}{\rho}\left(\boldsymbol{r}_{k}\right) \exp \left(\frac{-\left|\boldsymbol{r}_{k}-\boldsymbol{r}_{c}\right|^{2}}{2 R_{f}^{2}}\right) \\
& =v_{c} \sigma\left(M_{f}\right),
\end{aligned}
$$

where $\boldsymbol{r}_{c}=(L / 2, L / 2, L / 2), \sigma\left(M_{f}\right)$ is the rms level of fluctuations within a Guassian window of size $R_{f}=10 h_{50}^{-1} \mathrm{Mpc}$, and the sum in $\boldsymbol{k}$ is over all points in the lattice.
The simulations use a grid of size $N=16$, a practical limit for computations run in a small workstation environment such as a micro Vax II. In choosing the physical scale $L$ of the volume to simulate, there are competing factors to consider. One would like to use as large a volume as possible to improve the sampling of long-wavelength perturbations and correctly model tidal effects on matter late-infalling into the cluster. However, for a given $N$, increasing $L$ means degrading the small-scale resolution of the experiment. A value $L=50 h_{50}^{-1}$ $\mathrm{Mpc}$ is chosen as a compromise between these two constraints. On the small scale, this allows resolution of structure down to a limit of $\sim 200 h_{50}^{-1} \mathrm{kpc}$, while on the large scale, the rms fluctuations on the scale of the simulation cube $\left(M \sim 10^{16} h_{50}^{-1}\right.$ $\left.M_{\odot}\right)$ are still linear at the present epoch. Because of the nonzero expectation values of $\delta \rho / \rho$ over the entire volume, we allow for a DC component in the density field when generating the grid initial conditions. This means that the average density of the simulated volume will, in general, be greater than the mean cosmological background density.

The applied constraint only fixes the smoothed density at an arbitrary point in space, it does not directly constrain the properties of peaks in the density field. However, if the contrained density is sufficiently large, $v_{c} \gtrsim 3$, then it is likely that a peak will lie near the constrained point. The value of $v_{\mathrm{pk}}$ is determined directly from the realization by smoothing the field at all grid points and finding the maximum.

\section{c) N-Body + Hydrodynamic Evolution}

The Zel'dovich approximations is used to generate particle positions and velocities at an initial redshift $z_{i}=7$ from the density fields generated above (Efstathiou et al. 1985). The procedure displaces positions of an initial lattice of particles and assigns velocities in a manner consistent with linear theory growing modes of the generated density perturbations. The rms one-dimensional particle displacement was 0.2 grid cells at the initial epoch. Because the simulated volumes have some net positive overdensities $\delta_{i}$, the models are evolved using scale factors $R(t)$, consistent with background metrics of positive curvature. The mean recession velocities are reduced by an amount $1-2 \delta_{i} / 3$ in agreement with linear theory, implying a value of $\Omega_{i}=\left(1+\delta_{i}\right) /\left(1-2 \delta_{i} / 3\right)^{2}$ for the simulated volume. For the model studied here, $\delta_{i}=0.086$ and $\Omega_{i}=1.22$.

Two sets of 4096 particles are used in the simulations-one set to represent the collisionless dark matter, another to model the baryonic gas. Equal numbers of gas and dark particles are used with the latter 9 times as massive to give $\Omega_{\mathrm{ICM}}=0.1$. Each gas particle represents $m_{g}=2.1 \times 10^{11} h_{50}^{-1} M_{\odot}$. The distributions are coupled via gravity using the P3M method (Efstathiou and Eastwood 1981; Hockney and Eastwood 1985), and the gas physics of the baryons is followed using SPH (smoothed particle hydrodynamics - Gingold and Monaghan 1977; Monaghan 1985). The combined N-body and hydrodynamic method is described in a previous paper (Evrard 1988).

The particles representing the gas carry a local thermal energy $\epsilon_{i}=\left(k T_{i} / \mu m_{p}\right)$ and local densities $\rho_{i}$ at the location of particle $i$ are calculated by smoothing the nearby particle field

$$
\rho_{i}=m_{g} \sum_{j} W\left(r_{i j}, h_{i}\right),
$$

where

$$
W\left(r_{i j}, h_{i}\right)=\frac{1}{\pi^{3 / 2} h_{i}^{3}} e^{-r_{i j}{ }^{2} / h_{i}^{2}}
$$


is a Gaussian kernel which interpolates on a local smoothing scale $h_{i}$. The smoothing scale is allowed to vary both spatially and temporally to increase the dynamic range accessible to a given experiment.

The local pressure is described by an ideal $(\gamma=5 / 3)$ gas equation of state

$$
P_{i}=(\gamma-1) \epsilon_{i} \rho_{i}
$$

For simplicity, a fully ionized, primordial gas is assumed to hold throughout the evolution of the cluster implying a mean molecular weight $\mu=0.6$. Gas particles are accelerated by gravity and by pressure gradients measured via a local interpolation involving the gradient of the smoothing kernel. The gas force is written as an antisymmetric pairwise sum over neighboring particles to guarantee momentum and angular conservation to machine accuracy

$$
\left(\frac{\nabla P}{\rho}\right)_{i}=m_{g} \sum_{j}\left(\frac{P_{i}}{\rho_{i}^{2}}+\frac{P_{j}}{\rho_{j}^{2}}\right) \nabla W\left(r_{i j}, h_{i j}\right),
$$

where $h_{i j}=\frac{1}{2}\left(h_{i}+h_{j}\right)$. A particle's thermal energy changes due to $P d V$ work, shock heating, and radiative cooling, although the latter is ignored in these $16^{3}$ particle models, which lack resolution adequate to define cooling flows. Shock heating is incorporated using an artificial viscosity, which has the effect of increasing the pressure in strongly convergent flows from $P \propto c^{2}$ to $P \propto v^{2}$. Spherical cluster evolution (Evrard 1988) and planar shock tube experiments show that the form of the viscosity employed is effective in preventing interparticle penetration and satisfying shock jump conditions even in very strong shocks (Lattanzio et al. 1986). Heat conduction and any other energy transport or source mechanisms are not included in the calculation.

The smoothing length $h_{i}$ varies with local density $h_{i} \propto \rho_{i}^{-1 / 3}$ within lower and upper limits determined by the Courant condition and the range of the linked list in the short-range force calculation, respectively. The gravitational force is softened by using the potential $\phi(r) \sim\left(r^{2}+\epsilon^{2}\right)^{-1 / 2}$ with $\epsilon=150 h_{50}^{-1} \mathrm{kpc}$ kept fixed in the physical frame. The hydrodynamic resolution $h$ is dependent on the local density and temperature, but conditions are such that values $h \simeq 200 h_{50}^{-1} \mathrm{kpc} \sim \epsilon$ are reached in the highest density regions. The second form of artifical viscosity discussed in Evrard (1988) is used with coefficients $\alpha_{1}=0.5, \alpha_{2}=2.5^{1}$.

The initial temperature of the gas is arbitrarily taken to be $T_{i}=10^{4} \mathrm{~K}$. As will be demonstrated in $\S \mathrm{V}$, results are insensitive to the value of $T_{i}$ as long as the initial entropy $s_{i}=$ $\ln \left(T_{i} / \rho_{i}^{\gamma-1}\right)$ is small compared to the minimum final entropy. Time is used as the integration variable, with fixed time step $\delta t=1.9 \times 10^{7} h_{50}^{-1}$ yr corresponding to 700 steps in a Hubble time. Energy is conserved to better than one percent (in the sense discussed by Efstathiou et al. 1985), with momentum and angular momentum conservation much higher. The simulation presented here consumed $\sim 3$ microVax II CPU days. Table 1 provides a summary of various parameter values for the simulation.

Output files were dumped at 10 epochs between $z=7$ and $z=0$ equally spaced in time intervals of $1.3 h_{50}^{-1}$ billion years. In analyzing output, one often needs to compute simple inte-

\footnotetext{
${ }^{1}$ Note these coefficients are termed $\beta_{1}$ and $\beta_{2}$ in that paper; alpha is used here to minimize confusion with all the other betas in paper.
}

TABLE 1

\begin{tabular}{|c|c|}
\hline Parameter & Value \\
\hline 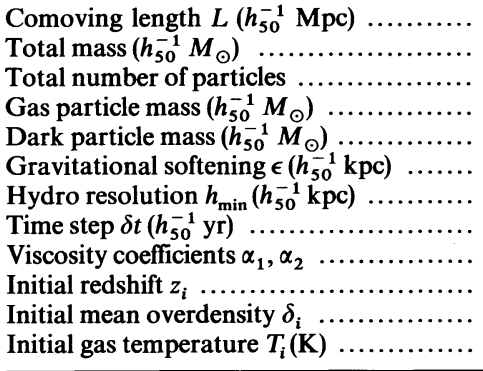 & $\begin{array}{l}50 \\
8.7 \times 10^{15} \\
8192 \\
2.1 \times 10^{11} \\
1.9 \times 10^{12} \\
150 \\
200 \\
1.9 \times 10^{7} \\
0.5,2.5 \\
7 \\
0.086 \\
10^{4}\end{array}$ \\
\hline
\end{tabular}

Summary OF MOdel Parameters

grals of the form

$$
Q=\int_{V} d^{3} x \rho(x) q(x) .
$$

For a density distribution represented by a set of discrete particles each of mass $m_{p}$, the density-weighted integral goes over to a sum over particles so that

$$
Q=m_{p} \sum_{i} q_{i}
$$

where $q_{i}$ is the value of the quantity at the location of particle $i$. In this way, calculation of the X-ray luminosities, surface brightness, the Sunyaev-Zel'dovich effect, etc., can be reduced to straightforward sums over the gas particle distribution.

\section{RESULTS}

\section{a) Evolution of the Cluster Volume}

The evolution of the gas and dark matter particles in a comoving $20 h_{50}^{-1} \mathrm{Mpc}$ cube centered on the present position of the cluster is shown Figure 1. The volume contains roughly half the mass in the simulation, about $4 \times 10^{15} h_{50}^{-1} M_{\odot}$ in total. At $z=1.62$, the cluster core is developing at the intersection of mildly nonlinear sheets. The cluster grows through accretion of surrounding material as well as through mergers with smaller, collapsed systems. The most obvious example of the latter is the satellite roughly one-fourth as massive as the main cluster component visible to the southeast at redshift $z=0.54$. At an epoch between $z=0.25$ and $z=0.15$, these two components merge to create the single dominant cluster seen at the present.

The distribution of gas particles in Figure 1 differs from that of the dark matter in two ways. First, the dark matter appears more "knotty" than the gas - small clumps of particles evident in the DM distribution are often absent in the gas. This effect is numerical in origin in that it is a direct manifestation of the discreteness limit of SPH. The algorithm attempts to smooth the hydrodynamics on a scale of 20 particles, which effectively prevents the formation of dense clumps containing only a few tens of particles. The other apparent difference in the distributions is the more rounded character of the gas at late times compared to the dark matter. This difference has a physical basis - the isotropic pressure tensor of the gas. The collisionless dark matter retains its memory of the merger event to the present epoch - witness the elongation of the DM particle distribution along the direction of merger. In contrast, the drive toward equipartition in the collisional gas component serves to 


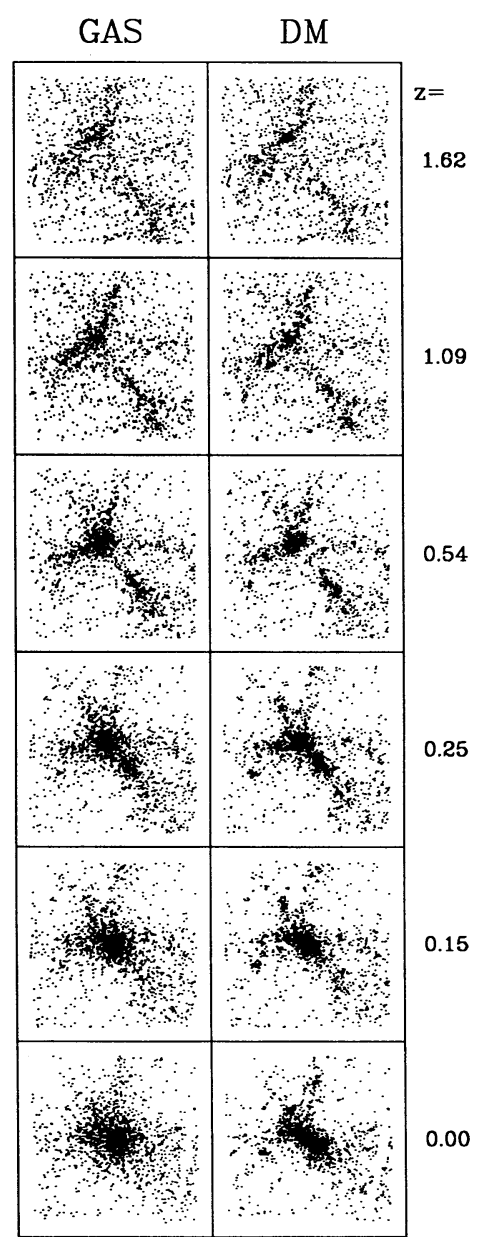

Fig. 1.-Evolution of the gas and dark matter (DM) particles within a comoving $20 h_{50}^{-1} \mathrm{Mpc}$ cube centered on the present cluster position. Redshifts are indicated to the right of the panels which show the distributions projected along the $z$-axis of the simulation. Note the more rounded character of the gas distribution at late times.

round out the gas distribution to resemble more closely the shape of the underlying, more spherical potential.

An attempt to view the gas in both real space and the thermodynamic space of particle density $n=\rho / \mu m_{p}$ and temperature $T$ is made in Figure 2. The gas particles in the same volume viewed in Figure 1 are shown binned in $n$ (Fig. $2 a$ ) and $T$ (Fig. $2 b$ ). Evolution proceeds in columns from left to right with density and temperature decreasing from upper (row I) to lower (row IV) panels in the figure. Density divisions occur at $10^{-3}, 10^{-4}$, and $10^{-5} h_{50}^{2} \mathrm{~cm}^{-3}$, and temperature cuts are made at $6,0.6$ and $0.06 \mathrm{keV}$. (Note: $10^{8} \mathrm{~K}=8.61 \mathrm{keV}$.) The number of particles falling in each bin is given within each panel. The present cosmological baryon density corresponds to a particle number density $n_{0}=5 \times 10^{-7}\left(\Omega_{b} / 0.1\right) h_{50}^{2} \mathrm{~cm}^{-3}$, so the particles in row I of Figure $2 a$ at $z=0$ are at a density contrast greater than 2000.

At early times, high-temperature gas is found only in small regions sufficiently overdense to have collapsed by that epoch. As the evolution proceeds, shock heating of the gas moves out to progressively lower densities and larger radii so that, by the present epoch, high-temperature gas can be found over a wide range of densities. The merger of the binary system at $z \simeq 0.2$ is clearly visible at moderate densities and temperatures (rows II). At $z \simeq 1$, about $10^{14} h_{50}^{-1} M_{\odot}$ of gas is heated above $0.6 \mathrm{keV}$ roughly the edge of the Einstein IPC bandpass. This mass grows by about a factor 4 to the present. The mass at densities above $10^{-4} h_{50}^{2} \mathrm{~cm}^{-3}$ increases by a factor $\sim 2$ from $z=1.09$ to $z=0.54$ and varies little from that epoch to the present.

The evolution of these mass filling factors is shown more clearly in Figure 3, where the baryonic mass with density greater than $n$ and temperature greater than $T$ is shown for the same volume and epochs viewed in Figures 1 and 2. Collapse of roughly $10^{13} h_{50}^{-1} M_{\odot}$ of gas at a density $n \sim 0.004 h_{50}^{2} \mathrm{~cm}^{-3}$ and temperature $T \sim 5 \times 10^{7} \mathrm{~K}$ is evident at $z \simeq 1$. This gas remains fairly unperturbed until the merger impact, which serves to increase the maximum density and temperature. The steep dependence of mass with temperature at late epochs indicates that the gas in the cluster is close to isothermal. Polytropic models, which have $T \propto n^{\gamma-1}$, are clearly not favored by the data in Figure 3. If the gas were polytropic, then the shapes of the density and temperature filling factors would be the same (within a scaling factor on the logarithmic axes) which is not the case in Figure 3. Since the physical mechanism responsible for heating the cluster gas, relaxation via shock heating, is not an adiabatic process, there is no underlying reason why polytropic models should describe well the present state of ICM.

\section{b) X-Ray Luminosity, Flux, and Surface Brightness Profiles}

The emissivity due to free-free encounters between electrons and ions in a fully ionized primordial plasma $(X=0.76$, $Y=0.24$ ) with temperature $T$ and particle density $n$ is given by (Spitzer 1968)

$$
\begin{aligned}
\epsilon_{v}=4.32 \times 10^{-28} n^{2} T^{1 / 2}(h / k T) \\
\times \bar{g}(h v / k T) e^{-h v / k T} \operatorname{ergs~s}^{-1} \mathrm{~cm}^{-3} \mathrm{~Hz}^{-1},
\end{aligned}
$$

where $\bar{g}(h v / k T)$ is an averaged Gaunt factor providing quantum mechanical corrections to the classical expression. Define a bolometric emissivity using unit Gaunt factor

$$
\epsilon_{\mathrm{bol}}=\int_{0}^{\infty} d v \epsilon_{v}=4.32 \times 10^{-28} n^{2} T^{1 / 2} \operatorname{ergs~s}^{-1} \mathrm{~cm}^{-3}
$$

Then the energy radiated within a given band $E_{1}-E_{2}$ can be expressed as

$$
\epsilon_{\mathrm{band}}=f_{\mathrm{band}}(T) \epsilon_{\mathrm{bol}}
$$

where

$$
f_{\text {band }}(T)=\int_{E_{1} / k T}^{E_{2} / k T} d \eta \bar{g}(\eta) e^{-\eta}
$$

The band limited $\mathrm{X}$-ray emission from a given volume $V$ is found by

$$
\begin{aligned}
L_{x} & =\int_{V} d^{3} x \epsilon_{\text {band }}(x) \\
& =4.32 \times 10^{-28} \int_{V} d^{3} x n^{2}(x) T^{1 / 2}(x) f_{\text {band }}[T(x)] \text { ergs s }^{-1} .
\end{aligned}
$$

Using the discrete information in the simulations, equation (11) implies the $\mathrm{X}$-ray luminosity from the volumes viewed in Figures 1 and 2 can be calculated by

$L_{x}=4.32 \times 10^{-28}\left(\mu m_{p}\right)^{-2} m_{g} \sum_{i} \rho_{i} T_{i}^{1 / 2} f_{\text {band }}\left(T_{i}\right) \operatorname{ergs~s}^{-1}$, 


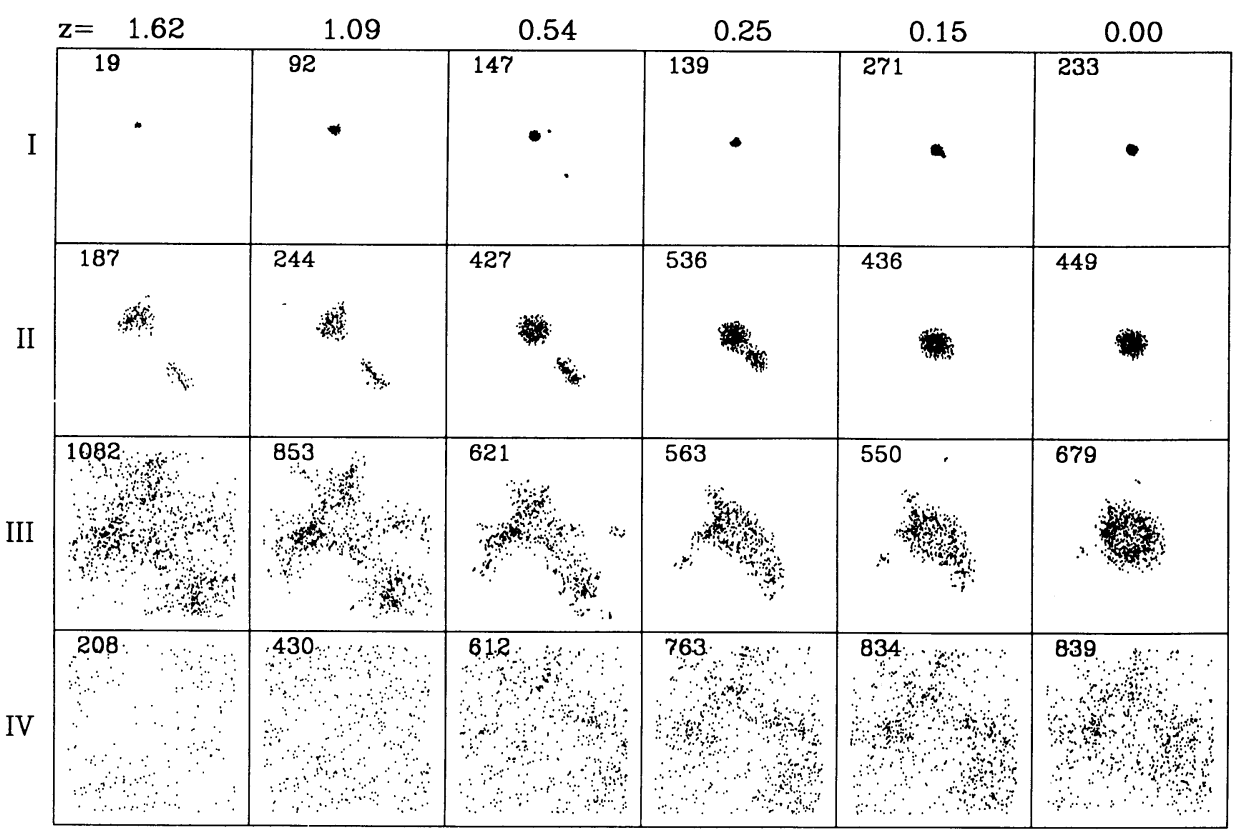

FIG. $2 a$

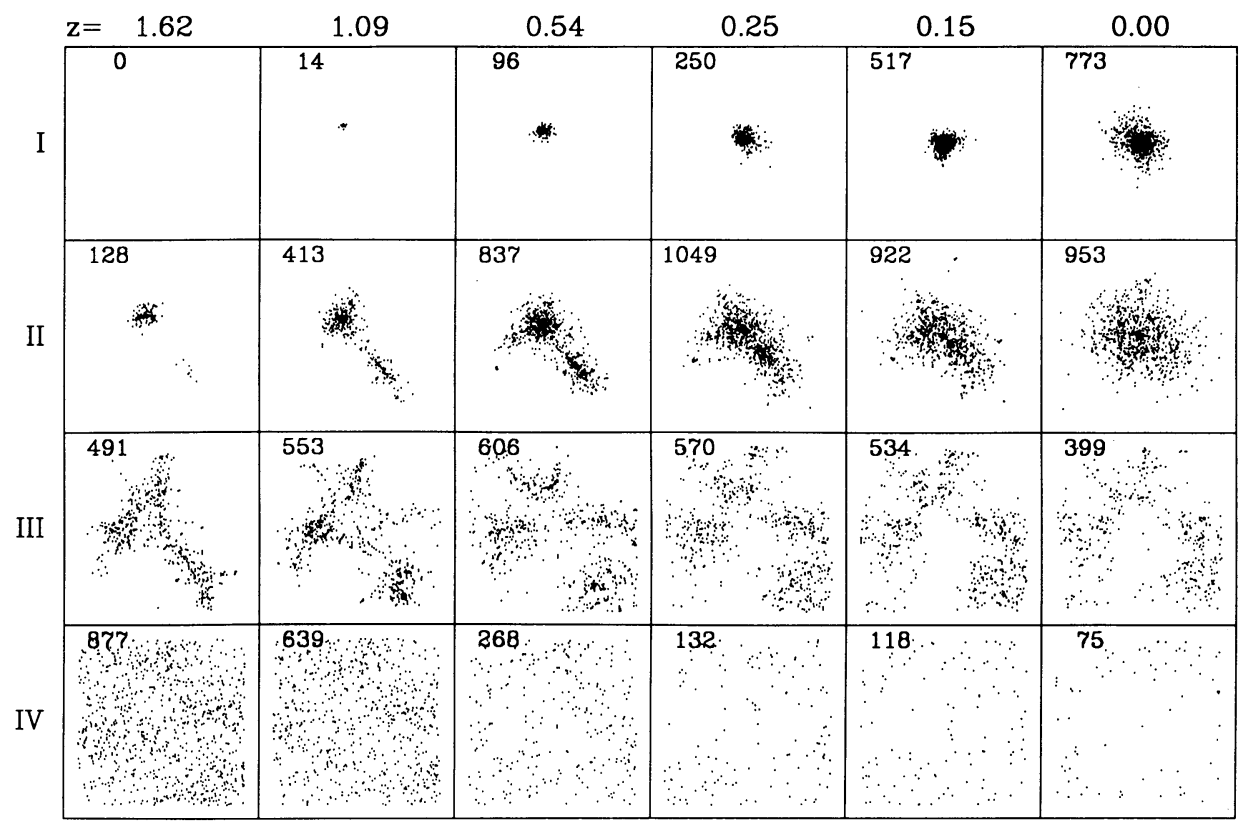

FIG. $2 b$

Fig. 2.- "Phase space" scatter plots showing the gas particles in the same volume as Fig. 1 but binned in density and temperature ranges: (a) bins in

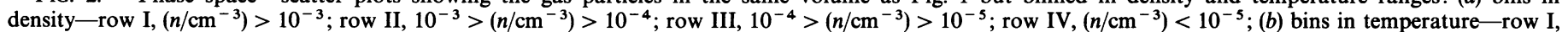

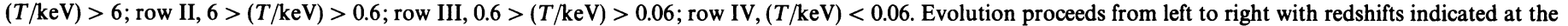
top of each column.

where the sum runs over particles within the volume. For the cluster viewed at redshift $z$, the flux $F_{x}$ of X-rays at Earth in an observed energy band $E_{1}-E_{2}$ is (Weinberg 1972)

$$
F_{x}=(4 \pi)^{-1}\left(\frac{H_{0}}{2 c}\right)^{2}\left\{\frac{L_{\text {bol }} f_{\text {band }}^{\prime}}{(1+z)^{2}\left[1-(1+z)^{-1 / 2}\right]^{2}}\right\} \text {, }
$$

where $f_{\text {band }}^{\prime}$ is the integral given by equation (15) over an appropriately redshifted energy range $E_{1}(1+z)$ to $E_{2}(1+z)$.
The evolution of the rest frame luminosities and observed flux in two energy bands is shown in Figure 4. Soft $0.5-3 \mathrm{keV}$ emission is given by the solid line, while the dashed line shows harder $2-10 \mathrm{keV}$ radiation. Gaunt factors were calculated using the Born approximation (Kellogg, Baldwin, and Koch 1975) which is accurate to within $\sim 10 \%$ of the exact calculations (Karzas and Latter 1961) for the energies and temperatures considered here. Galactic absorption is ignored in computation of the incident flux. The solid diamonds in the 


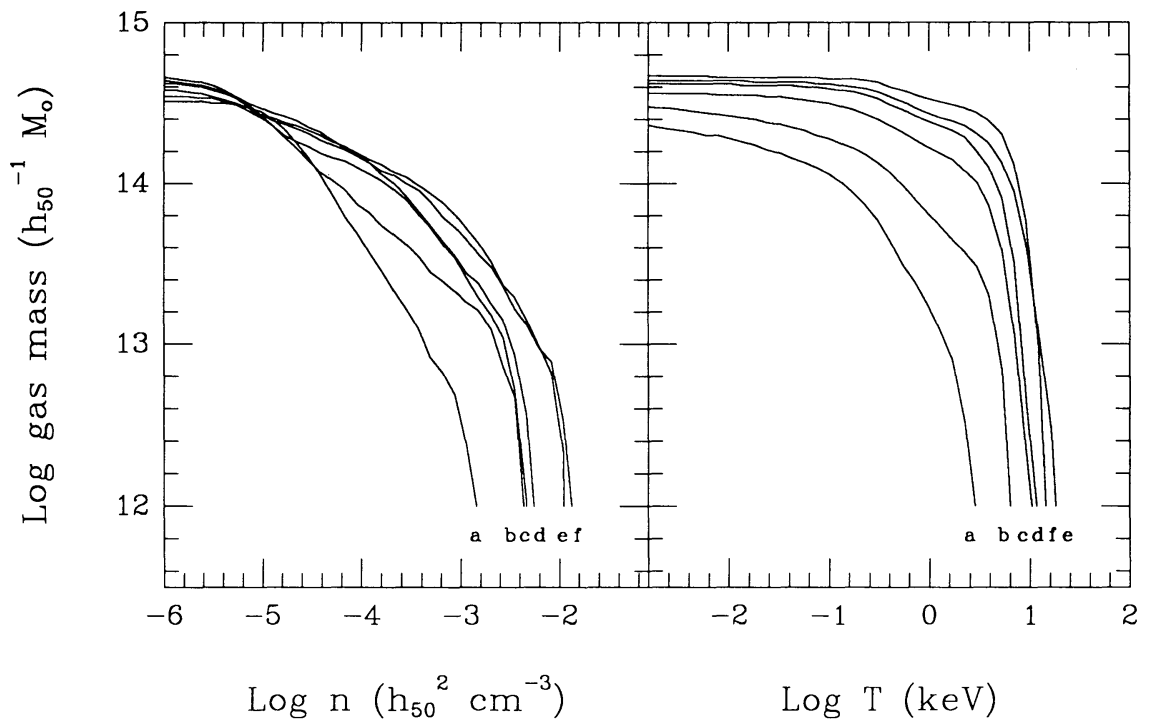

Fig. 3.-Amount of mass above density $n$ and temperature $T$ for the volume viewed in Figs. 1 and 2. Lines correspond to different redshifts: a, $z=1.62$; $b$, $z=1.09 ; \mathrm{c}, z=0.54 ; \mathrm{d}, z=0.25 ; \mathrm{e}, z=0.15 ; \mathrm{f}, z=0$. The steep dependence of mass with temperature at late times indicates the cluster is close to isothermal. Polytropic models $T \propto n^{\nu-1}$ are not favored by these data, since the shapes of the density and temperature curves are dissimilar.

figure indicate the observed flux and inferred luminosity of the Coma cluster in the $2-10 \mathrm{keV}$ band (Lahav et al. 1989).

The cluster intrinsic luminosity grows rapidly as the first resolved structure collapses between redshifts $z=1.6$ and $z=1.1$. The rapid rise in luminosity in this era is due to dynamic range limitations and should not be considered physical. Higher resolution experiments are needed to resolve emis-

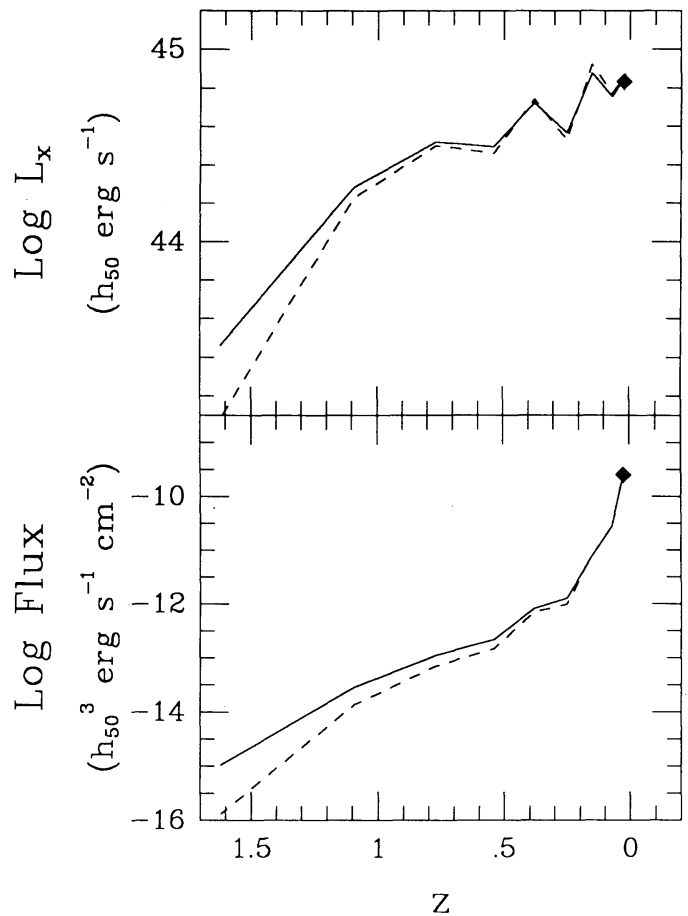

Fig. 4.-Evolution of the cluster rest frame luminosity and observed flux The solid line shows soft $0.5-3 \mathrm{keV}$ radiation, while the dashed line is for hard $2-10 \mathrm{keV}$ radiation. The cluster luminosity grows by an overall factor $\sim 3$ from redshifts $z=1$ to $z=0$, with the growth being punctuated by mergers of surrounding satellites onto the parent cluster. sion from the characteristically smaller mass scales collapsing at redshifts $z \gtrsim 1$. From $z=1.1$ to the present, the luminosity increases more slowly, with an $e$-fold increase occurring from roughly $z=0.7$ to the present, a time scale of $\sim 8 h_{50}^{-1}$ billion years. More importantly, the evolution is not a simple monotonic function of time; rather, the growth is punctuated by accretion/merger events of the parent cluster with smaller surrounding satellites. The merger of the two dominant components at redshift $z \simeq 0.2$ increase the luminosity by a factor 2.5. Note that the luminosity can decrease after such disturbances - evidence that the central density is varying due to oscillations or sonic waves generated by the merger event.

The point of view that a cluster simply appears at some collapse redshift with a well-defined and thereafter constant $\mathrm{X}$-ray luminosity is clearly an oversimplification of the true physical situation. At no time in the evolution is an exact steady state achieved, since accretion of infalling material and tidal interactions with neighboring systems are continually at work to disturb a cluster's equilibrium. However, despite these external influences, the gas within the shock front is fairly close to hydrostatic equilibrium, as is shown in $\S$ III $e$, below.

The flux which would be observed at Earth from this cluster placed at the distance of Coma $(z=0.023)$ is comparable to that observed from Coma by EXOSAT $F_{x}=3.2 \times 10^{-10}$ ergs $\mathrm{s}^{-1} \mathrm{~cm}^{-2}$ (Lahav et al. 1989). The flux drops over two orders of magnitude as the look-back epoch increases to redshift $z=0.25$. This cluster would fall below the Einstein Medium Sensitivity Survey flux limit of $2.6 \times 10^{-14} \mathrm{ergs} \mathrm{s}^{-1} \mathrm{~cm}^{-2}$ at a redshift $z \simeq 1$.1. However, detection at this limit requires that the cluster emission not extend over an area much larger than $1 \operatorname{arcmin}^{2}$. Figure 5 presents surface brightness maps of the cluster at redshifts ranging from $z=1.1$ to the distance of Coma. The maps at high redshift are shown at a resolution sharper than that of the Einstein IPC. The map at $z=1.09$ is smoothed with a Gaussian filter with half-power radius $\sigma_{h}=$ 0.25 , while those at $z=0.54,0.25$, and 0.15 are smoothed on a scale $\sigma_{h}=0.5$, the expected resolution of the PSPC aboard the ROSAT satellite. The map at $z=0.07$ is at a resolution compa- 


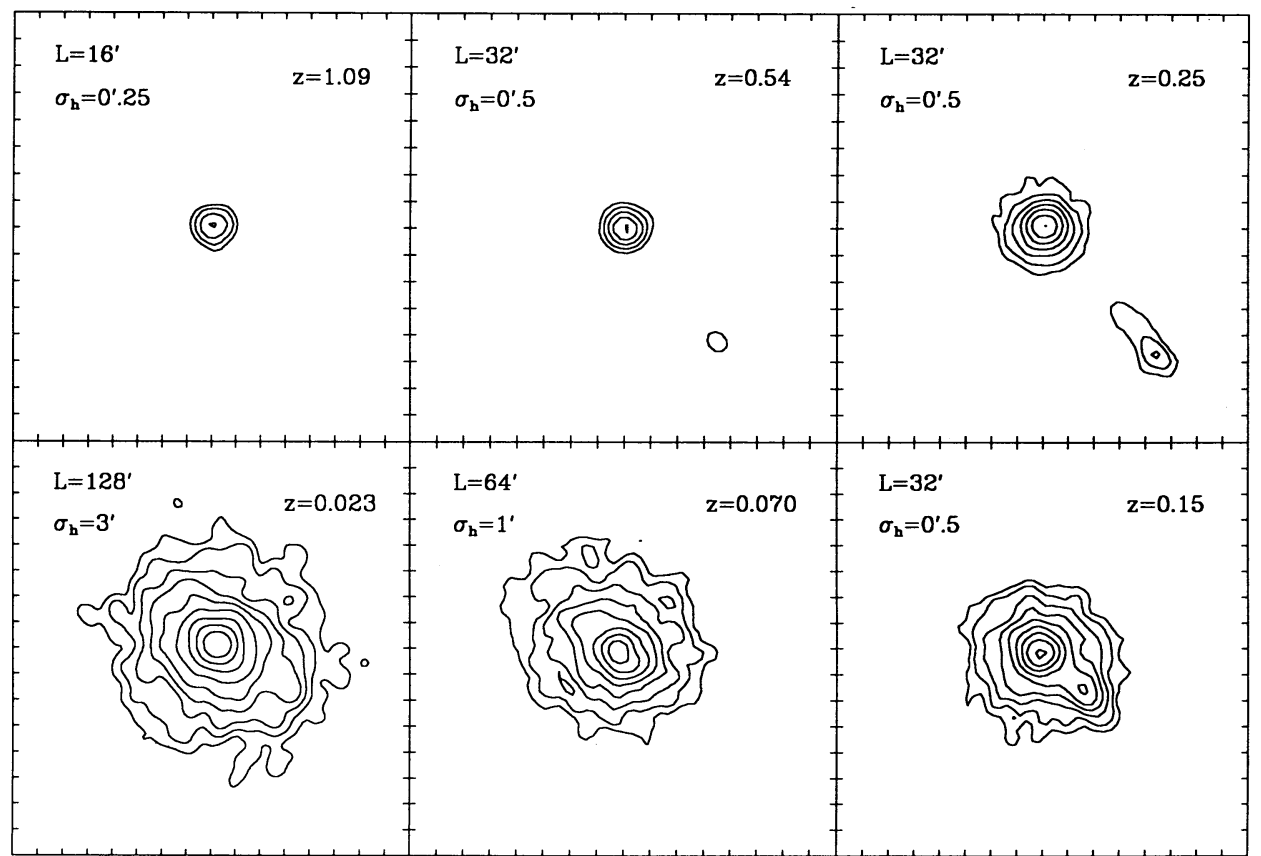

FIG. 5.- The cluster X-ray emission in the Einstein $0.5-3 \mathrm{keV}$ band seen at different look-back epochs. The surface brightness of the model is binned in projection and smoothed using a Gaussian filter with half-power radius $\sigma_{h}$. Evolution proceeds clockwise from the upper left with the viewing redshift, size of window $L$, and smoothing scale $\sigma_{h}$ indicated within each panel. The lowest contour is at $10^{-4} h_{50}^{3}$ IPC count s ${ }^{-1}$; contours are spaced by factors of $10^{1 / 3}=2.15$.

rable to the IPC, $\sigma_{h}=1^{\prime}$, while discreteness limitations force the map at $z=0.023$ to be smoothed on a scale $\sigma_{h}=3^{\prime}$. All fluxes have been converted to Einstein IPC count rates using a simple conversion factor (Giacconi et al. 1979)

$$
1 \text { IPC counts s}{ }^{-1} \operatorname{arcmin}^{-2}=2.5 \times 10^{-11} \mathrm{ergs} \mathrm{s}^{-1} \mathrm{~cm}^{-2} \text {. }
$$

The size of the viewing window $L$ ranges from roughly onefourth the IPC field of view at $z=1.09$ to twice the IPC field at $z=0.023$. The lowest contour shown is $10^{-4} h_{50}^{3}$ counts $\mathrm{s}^{-1}$; contours are spaced by factors of 2.15 , corresponding to three levels per decade of flux. No background has been added to these pictures.

At $z=0.15$, the merging satellite clearly distorts the isophotes at levels 0.002 counts $\mathrm{s}^{-1}$ in the direction of impact. At $z=0.07$, the distortion is still evident but in the opposite direction. The change in isophotal pointing is due to the momentum absorbed by the parent cluster from the captured satellite. Viewed at the distance of Coma, the cluster isophotes display general features common to those seen in Coma (Johnson et al. 1979) and A2256 (Fabricant, Kent, and Kurtz 1989). All have roughly elliptical isophotes with indications of boxiness, twists, and some jitter in the isophotal centers.

Radial profiles of these projected surface brightness maps are shown in Figure 6. The profiles are fit to the form

$$
\Sigma_{x}(\theta)=\Sigma_{0}\left[1+\left(\theta / \theta_{x}\right)^{2}\right]^{-3 \beta_{\mathrm{fit}}+1 / 2} .
$$

Values of the central surface brightness $\Sigma_{0}$, X-ray core angular size $\theta_{x}$, and exponent $\beta_{\text {fit }}$ for each viewing epoch are given in Table 2 . The central surface brightness and angular core radius grow with time. The cluster would be essentially pointlike to Einstein beyond a redshift $z \simeq 0.5$. The exponent $\beta_{\text {fit }}$ governing the outer profile is fairly constant at moderate to low redshifts.
The larger values inferred at high redshift are poorly determined since the fits extend to only $\sim 2$ core radii. The value of $\beta_{\mathrm{fit}}=0.76 \pm 0.02$ seen at $z=0.023$ is very similar to the inferred value for Coma $\beta_{\mathrm{fit}}=0.75 \pm 0.02$ (Hughes 1989). With the assumption of an isothermal temperature distribution, this implies the spatial density of the ICM falls with radius as $n(r) \propto r^{-3 \beta_{\text {fit }}} \sim r^{-2.2}$ in the outer parts of the cluster. This is in good agreement with the true gas density profile examined in $\S$ III $c$, below.

\section{c) The Sunyaev-Zel'dovich Effect}

Another observable effect resulting from the presence of a hot ICM in clusters is the diminution of flux in the microwave

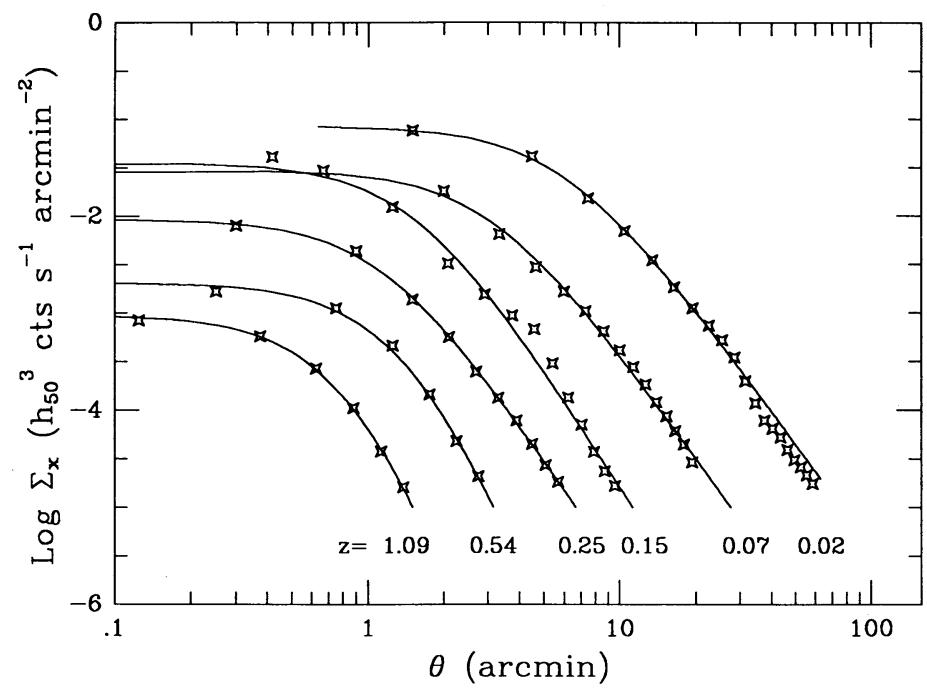

Fig. 6.-Radial profiles of the emission shown in Fig. 5 with fits to the isothermal $\beta$-model. Parameter values for the fits are given in Table 2. 
TABLE 2

Fits to Surface Brightness Profiles

\begin{tabular}{ccccc}
\hline \hline & \multicolumn{2}{c}{$\Sigma_{0}$} \\
$z$ & $\left(10^{-3}\right.$ counts s $\left.^{-1}\right)$ & $\theta_{x}$ & $\beta_{\text {fit }}$ \\
\hline $1.09 \ldots \ldots \ldots \ldots$ & $1.0 \pm 0.2$ & $1.0 \pm 0.3$ & $1.37 \pm 0.23$ \\
$0.54 \ldots \ldots \ldots \ldots$ & $2.0 \pm 0.4$ & $1.5 \pm 0.3$ & $1.23 \pm 0.17$ \\
$0.25 \ldots \ldots \ldots \ldots$ & $10 \pm 2$ & $1.2 \pm 0.3$ & $0.81 \pm 0.05$ \\
$0.15 \ldots \ldots \ldots \ldots$ & $36 \pm 10$ & $1.5 \pm 0.2$ & $0.85 \pm 0.04$ \\
$0.070 \ldots \ldots \ldots \ldots$ & $30 \pm 10$ & $3.1 \pm 0.4$ & $0.78 \pm 0.03$ \\
$0.023 \ldots \ldots \ldots \ldots$ & $84 \pm 5$ & $6.1 \pm 0.3$ & $0.76 \pm 0.02$ \\
\hline
\end{tabular}

background in the Rayleigh-Jeans region of the spectrum due to inverse Compton scattering of low-energy photons off hot plasma electrons (Zel'dovich and Sunyaev 1969; Sunyaev and Zel'dovich 1970). The predicted diminution in radiation temperature $T_{r}$ is

$$
\frac{\Delta T_{r}}{T_{r}}=-2 \int d l \sigma_{\mathrm{T}} n_{e} \frac{k T_{e}}{m_{e} c^{2}},
$$

where $n_{e}, T_{e}$, and $m_{e}$ are the electron number density, temperature, and mass; $\sigma_{\mathrm{T}}$ is the Thomson scattering cross section; and the integral is performed along a given line of sight. Observations of this effect are difficult and to date, only a few robust detections presently exist (Uson and Wildinson 1988; Birkinshaw 1989) at measured amplitudes of around $\Delta T \simeq 0.5 \mathrm{mK}$.

The expected decrements for the model cluster at different look-back times are contour mapped in Figure 7. The data have been smoothed using a Gaussian filter with a half-power radius of $1^{\prime}$, except for $z=0.023$ which was smoothed on a scale of $3^{\prime}$. Contour levels start at $0.1 \mathrm{mK}$ and are spaced by factors of $10^{0.2}$.

The cluster decrement is below $0.1 \mathrm{mK}$ with this resolution at $z=1.09$, but a value of $0.3 \mathrm{mK}$ would be expected with a smaller beam of 0.25 . At $z=0.54$, a $0.1 \mathrm{mK}$ decrement would be expected from an experiment with $1^{\prime}$ resolution. The central decrement increases to $0.4 \mathrm{mK}$ after the merger event at $z \simeq 0.2$ and remains roughly constant to the present. Viewed at the Coma distance, a decrement $\Delta T \simeq 0.5 \mathrm{mK}$ is present over a central $5^{\prime}$ region The profile falls off roughly as $\Delta T(\theta) \propto \theta^{-2}$ beyond $20^{\prime}$ at this epoch. Obtaining a clean reference signal to detect the central decrement would require a beam throw of $\gtrsim 40^{\prime}$.

\section{d) Profiles of the ICM}

Given the particle distribution at any redshift, the cluster proper is defined using a group finding algorithm based on joining sets of pairs with common members (a "friends-offriends" algorithm). The pairs are chosen to have separations less than 0.1 times the mean interparticle spacing. The center of the cluster is defined to coincide with the position of the most bound particle in the group. With the cluster center so defined, radial profiles of the cluster can be measured directly at each redshift.

Figure 8 shows profiles of density, temperature, radial velocity, enclosed mass, pressure and entropy for the gas at redshifts $z=1.09,0.54,0.25$, and 0.0 . Physical, not comoving, radii are plotted along the abscissa. The profiles are truncated at radii at which the total mean interior density is equal to 10 times the background value at that redshift.

At $z=1.09$, infall velocities of $500 \mathrm{~km} \mathrm{~s}^{-1}$ are evident at radii $r=1-2 h_{50}^{-1} \mathrm{Mpc}$ onto roughly $4 \times 10^{13} h_{50}^{-1} M_{\odot}$ of gas at a temperature $T=5 \times 10^{7} \mathrm{~K}$. The infall velocity increases to $800 \mathrm{~km} \mathrm{~s}^{-1}$ and the post shock temperatures approach $\sim 10^{8} \mathrm{~K}$ by $z=0.25$. This steepening of the shock strength generates a temperature profile at $z=0$ with a modest positive temperature gradient $T(r) \propto r^{0.3}$ within $1 h_{50}^{-1} \mathrm{Mpc}$ of the center. A decrease of the same order $T(r) \propto r^{-0.3}$ follows out to an Abell radius $R_{\mathrm{A}}=3 h_{50}^{-1} \mathrm{Mpc}$ with a sharper drop beyond as one

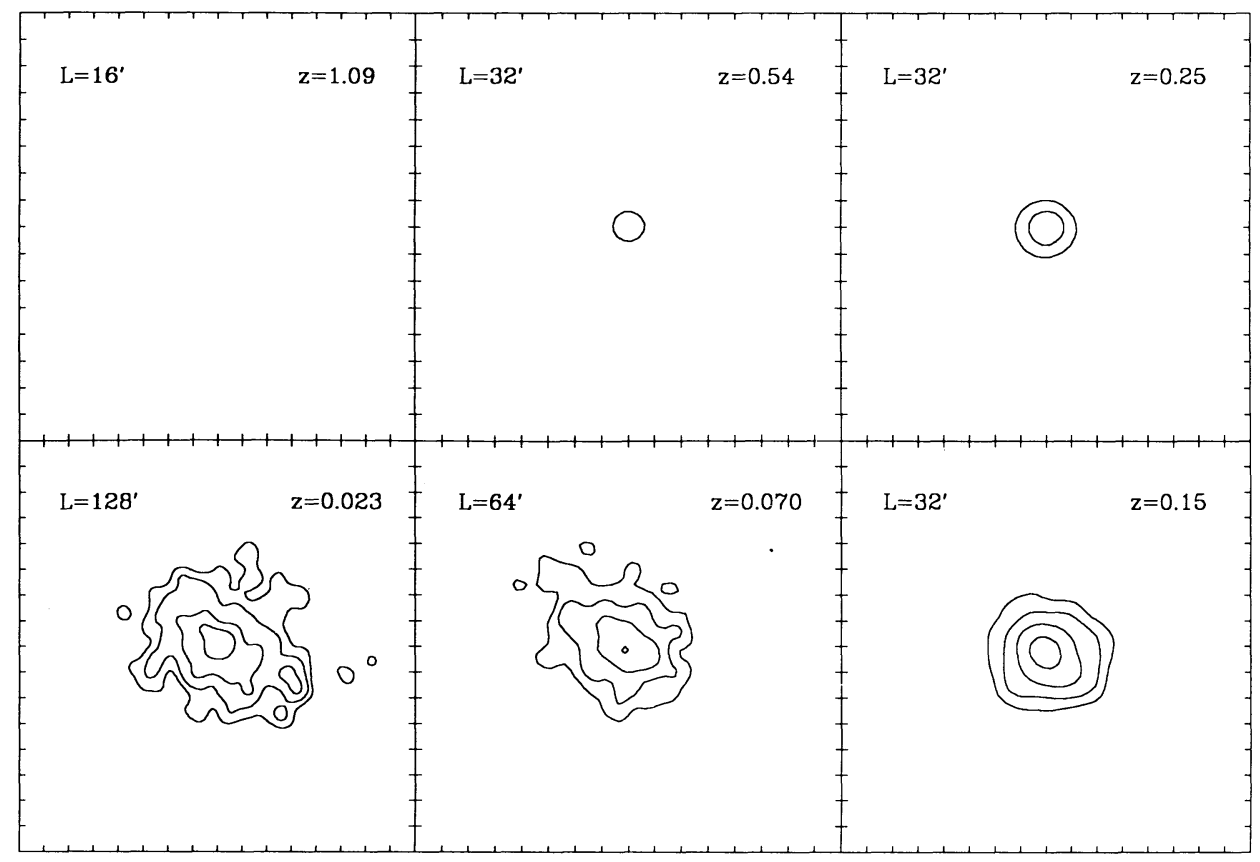

Fig. 7.-Maps of the Sunyaev-Zel'dovich decrement $\Delta T$ expected from the simulated cluster at different look-back epochs. The expected decrement is smoothed with a Gaussian having half-power radius $\sigma_{h}=1^{\prime}$ at all redshifts except $z=0.023$ which has $\sigma_{h}=3^{\prime}$. Format is otherwise identical to that of Fig. 5. Contour levels start at $0.1 \mathrm{mK}$ and are spaced by factors of $10^{0.2}=1.58$. 

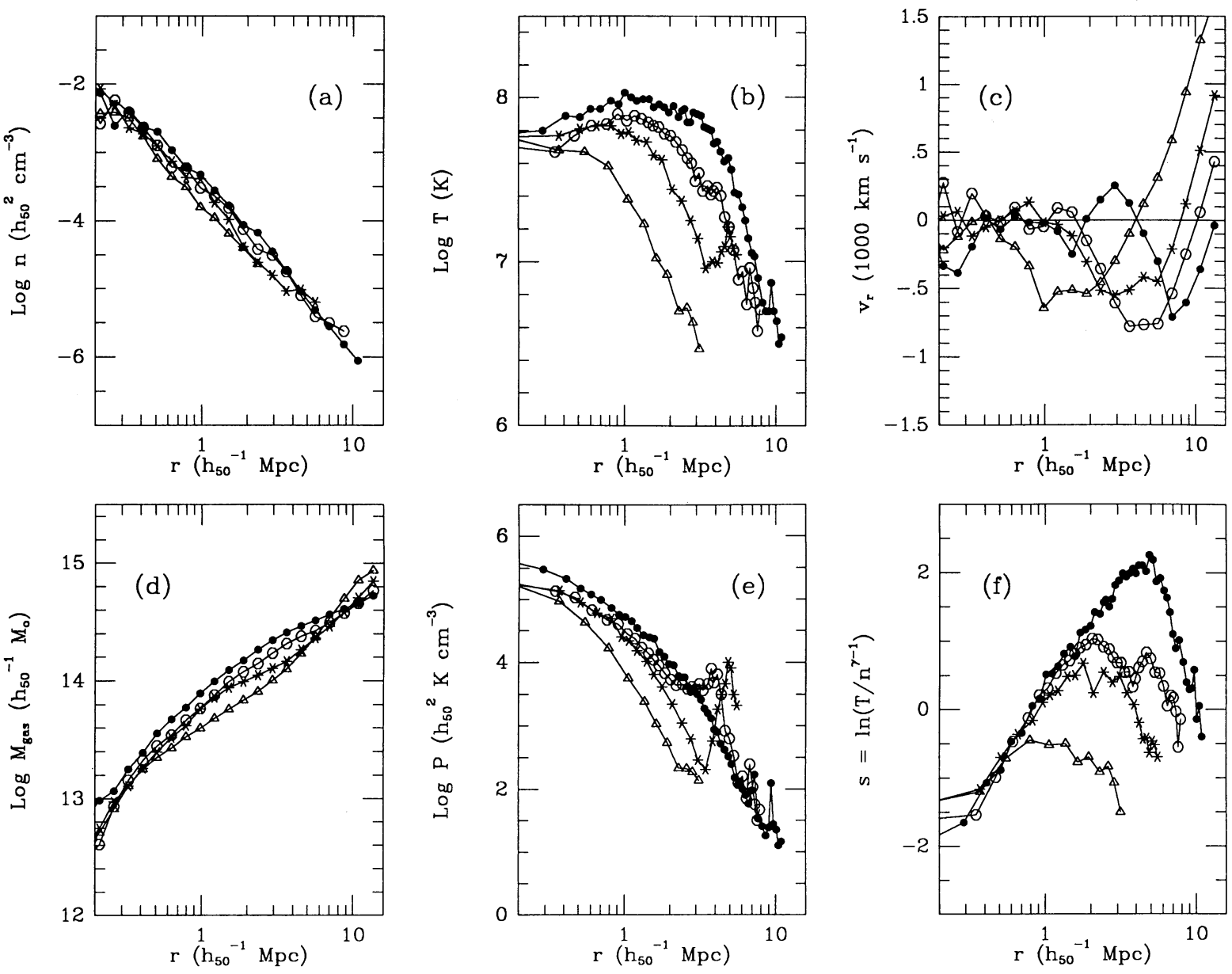

Fig. 8. - Radial gas profiles for the cluster at different epochs showing $(a)$ particle density, $(b)$ temperature, $(c)$ radial velocity, $(d)$ enclosed mass, $(e)$ pressure, and $(f)$ entropy. The different symbols correspond to different redshifts: $z=1.09$, open triangles; $z=0.54$, asterisks; $z=0.25$, open circles; and $z=0$, filled circles.

reaches the infall regime dominated by cooler, weakly shocked gas. A central temperature inversion was also exhibited by the one-dimensional infall models of Perrenod (1978) and appears to be a consequence of the fact that gas collapsing early is mildly shocked relative to late-infalling material.

The present density distribution does not exhibit an obvious core radius within which the density rolls over to a constant value. However, neither is the density run a pure power law. Instead, the $\log$ arithmic slope $d \log \rho / d \log r$ is shallower in the center, with a value $\sim-1.5$ inside $500 \mathrm{kpc}$, than in the outer parts where $d \log \rho / d \log r \sim-2.2$ at radii $r \sim 2 h_{50}^{-1} \mathrm{Mpc}$. Similar behavior is seen in the observed run of galaxy density with radius in clusters, although the observed profiles are sensitive to the choice of cluster center (Beers and Tonry 1986; Merrifield and Kent 1989). Unfortunately, since the X-ray emission is proportional to the square of the density, the density run in the center can play a significant role in determining the overall luminosity of the cluster. Indeed, the total luminosity formally diverges for radial gas profiles steeper than $\rho(r) \propto r^{-1.5}$. It is clear that at some level, extra gas physics beyond $P d V$ work and shock heating incorporated here will be important to the gas thermodynamics. Radiative cooling, feedback from star-forming regions, conduction, magnetic fields, and heating by galaxy motions all may play a role in determining the structure and emission from the inner few $100 \mathrm{kpc}$ of the cluster. Investigating these effects in detail is beyond the scope of the present paper, but some preliminary results for gas cooling are discussed in $\S \mathrm{V}$. The total luminosity of the simulated cluster is therefore uncertain because of the present poor understanding of the important physics affecting only the inner $\sim 5 \%$ of the total gas mass within an Abell radius.

The formation of the hot ICM can be viewed crudely as resulting from a spherically symmetric shock wave moving outward (in both an Eulerian and a Lagrangian sense) into accreting material. We can locate the approximate position of the shock by finding the maximum in the entropy profiles in Figure 8f. The maximum moves from $\sim 1 h_{50}^{-1} \mathrm{Mpc}$ to $5 h_{50}^{-1}$ $\mathrm{Mpc}$ in the roughly 10 billion years between $z=1.09$ and $z=0$, indicating the shock front propagates outward with a mean velocity of around $400 \mathrm{~km} \mathrm{~s}^{-1}$. The formation process leaves behind an entropy profile increasing radially as $s \propto 2.7 \ln (r)$.

The shock heating process is not $100 \%$ efficient in converting the kinetic energy of infall into heat. Subsonic gas velocities of around $\sigma_{\mathrm{gas}} \sim 300-400 \mathrm{~km} \mathrm{~s}^{-1}$ (one-dimensional) are found in the postshock regions. Again, Perrenod (1978) found similar behavior in his models. At $z=0$, the ratio of specific energy in these motions to thermal energy within an Abell radius is small, but nonzero $\sigma_{\text {gas }}^{2} /\left(k T / \mu m_{p}\right) \simeq 0.13$, where $\sigma_{\text {gas }}$ is the onedimensional velocity dispersion of the intracluster gas. 

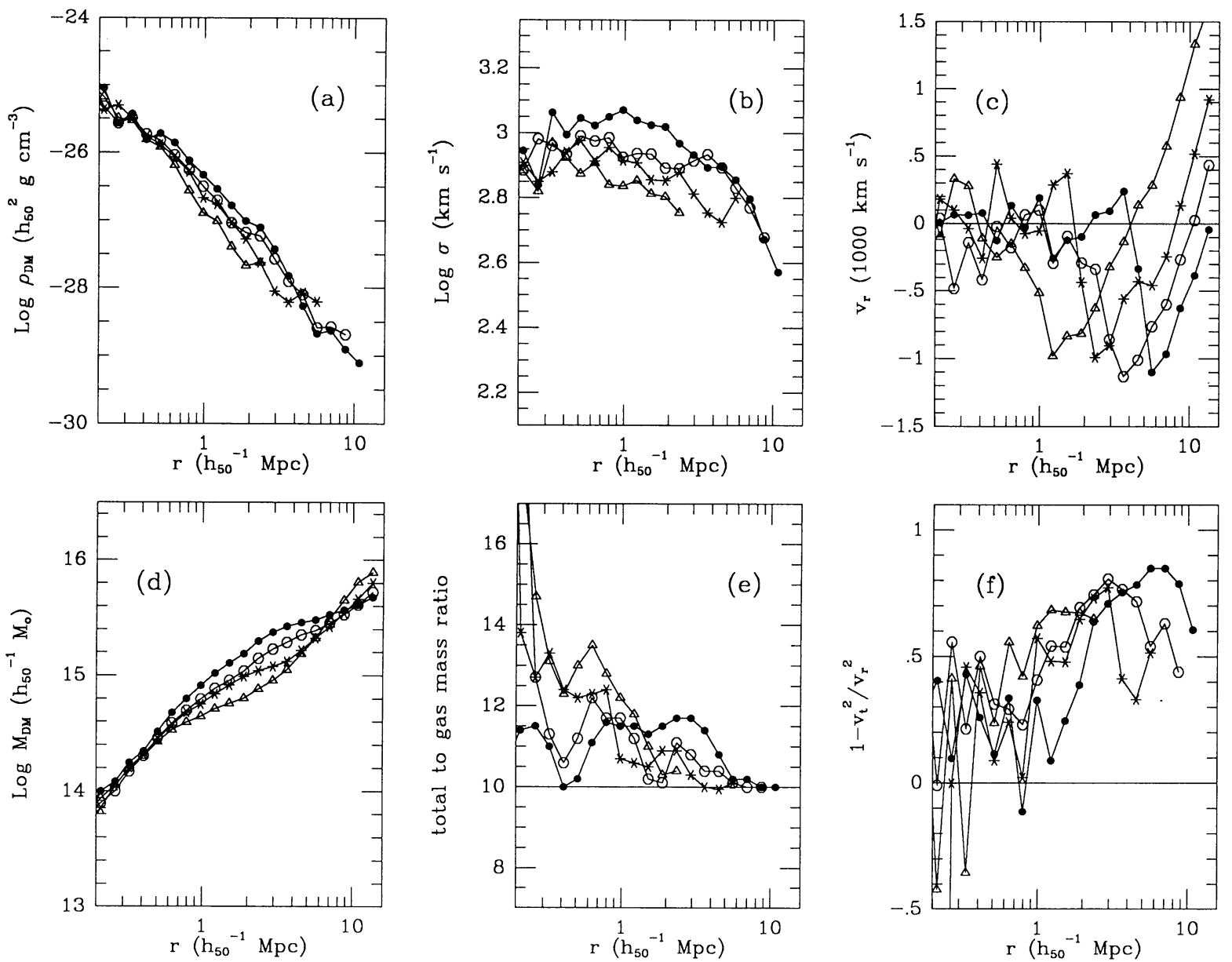

Fig. 9.-Radial profile for the dark matter in the cluster at different epochs showing $(a)$ density, $(b)$ velocity dispersion, $(c)$ radial velocity, $(d)$ enclosed mass, $(e)$ differential total-to-gas mass ratio, and $(f)$ velocity anisotropy. The different symbols correspond to same redshifts as in Fig. 8 .

\section{e) Evolution of the Dark Matter}

The evolution of the dark matter distribution is shown in Figure 9. The density profile evolves in a way similar to that of the gas. The degree of segregation of the two components is shown in Figure 9e, which displays the local total-to-gas mass ratio in spherical shells as a function of radius. If no segregation occurs, this ratio will equal the global value of $\Omega_{\mathrm{ICM}}^{-1}=10$. At early times, the artificial viscosity active in the shock front acts to prevent gas particle penetration through the cluster center while the collisionless dark matter is allowed to freestream. This results in roughly a $20 \%-30 \%$ enhancement of the dark matter density in the inner $1 h_{50}^{-1} \mathrm{Mpc}$. As the system evolves, this effect persists but at a lower amplitude of $10 \%-$ $20 \%$. Given this small magnitude, it is fair to say that no significant segregation of the two components occurs. However, the direction of the effect is always the same- the cluster total-to-baryon mass ratio is always slightly enhanced over the global value. This statement is not likely to apply at radii $\lesssim 100 h_{50}^{-1} \mathrm{kpc}$, where removal of pressure support through radiative cooling of the gas could serve to enhance the baryonic density relative to the dark matter.

The velocity dispersion of the dark matter increases slightly with time from $900 \mathrm{~km} \mathrm{~s}^{-1}$ at $z=1.09$ to $1150 \mathrm{~km} \mathrm{~s}^{-1}$ at the present epoch. Like the gas temperature, the velocity dispersion at $z=0$ shows modest variation with radius, rising gently to a maximum at $r \simeq 1 h_{50}^{-1} \mathrm{Mpc}$ and falling beyond this radius.
Unlike the temperature, the dispersion does not drop off more sharply at radii outside the relaxed portion of the cluster $r \gtrsim$ $4 h_{50}^{-1} \mathrm{Mpc}$. This is because the dispersion is conventionally defined relative to the center of mass velocity of the cluster, so bulk inflow at large radii is included in the estimate. Figure $9 c$ shows that infall velocities of $1000 \mathrm{~km} \mathrm{~s}^{-1}$ are common, higher than that found for the gas. The deceleration and shock heating of the gas as it hits the hot ICM are responsible for this difference - the collisionless dark matter is able to fall deeper into the potential well of the cluster, thereby generating larger radial velocities.

The anisotropy of the dark matter velocity field is displayed in Figure $9 f$, which shows the anisotropy parameter

$$
A(r)=1-v_{t}^{2}(r) / v_{r}^{2}(r)
$$

with $v_{r}(r)$ the radial and $v_{t}(r)$ the one-dimensional tangential components of the velocity at radius $r$. Radial orbits are clearly dominant in the outer parts of the cluster where $v_{r}^{2} \simeq 4 v_{t}^{2}$. Within the inner $1 h_{50}^{-1} \mathrm{Mpc}$ at present, the orbits are more nearly isotropic but retain a radial preference with $A \simeq 0.2$.

\section{f) Hydrostatic Equilibrium, Mass Modeling, and the " $\beta$-Discrepancy"}

Mass estimates of clusters from X-ray observations have commonly been based on the hydrostatic, isothermal $\beta$-model 
(Cavaliere and Fusco-Fermiano 1976; Bahcall and Sarazin 1977; Jones and Forman 1984; Hughes 1989; Fabricant, Kent, and Kurtz 1989). The model assumes that both the gas and galaxies in a cluster are isothermal and in hydrostatic equilibrium within a common potential. The form of binding mass profile which generates the potential is generally taken to be a King model approximation to an isothermal sphere

$$
\rho_{b}(r)=\rho_{b, 0}\left[1+\left(r / r_{c}\right)^{2}\right]^{-3 / 2},
$$

where the central density $\rho_{b, 0}$, core radius $r_{c}$, and velocity dispersion $\sigma$ are related by $\sigma^{2}=4 \pi G \rho_{b, 0} r_{c}^{2} / 9$. With this approximation and the assumption of hydrostatic equilibrium

$$
\frac{d \ln \rho_{g}(r)}{d \ln r}=-\frac{\mu m_{p}}{k T} \frac{M_{b}(r)}{r},
$$

the gas density then follows the form given by equation (1), where the power-law exponent $\beta$ is determined by ratio of specific energies $\beta \equiv \sigma^{2} /\left(k T / \mu m_{p}\right)$.

The numerical simulations provide an ideal forum for investigating the source of the $\beta$-discrepancy outlined in the introduction. The underlying assumptions of the isothermal $\beta$-model can be checked and the relevant observational quantities calculated. However, since galaxies are not explicitly included in the calculation, an added assumption must be made that galaxy trajectories sample fairly the dark matter orbits in the cluster. This assumption is valid as long as secondary dynamical affects such as dynamical fraction do not significantly affect the velocity dispersion of cluster galaxies.

As a starting point, we can address the question of whether the gas is in hydrostatic equilibrium. The radial velocities of the gas at $z=0$ shown in Figure $8 c$ exhibit no significant net inflow or outflow within $\sim 2 h_{50}^{-1} \mathrm{Mpc}$. This indicates that the gas within this radius is in hydrostatic equilibrium. As a further check, we can directly measure the mean acceleration experienced by mass elements in radial shells a distance $r$ from the cluster center. Figure 10 shows various components of the measured acceleration times radius. Line 1 gives the magnitude

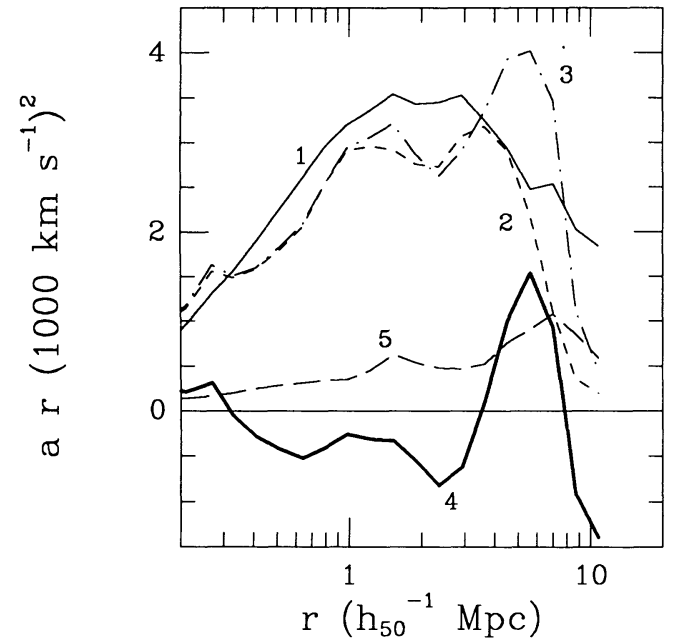

Fig. 10.-Components of the mean acceleration $a$ experienced by particles at radius $r$ express as the product $a r$. Different line types denote different components: line 1 (solid), magnitude of the radial gravitational component; line 2 (dashed), radial pressure gradient; line 3 (dot-dashed), radial pressure gradient including artificial pressure; line 4 (heavy solid), total radial acceleration; line 5 (long dashed), mean tangential acceleration. of the radial component of gravity, line 2 gives the mean pressure gradient, line 3 is the pressure gradient including the effects of the artificial viscosity, and line 4 is the total radial component of the acceleration (line 3 minus line 1). Line 5 gives the mean tangential acceleration as a function of $r$. Note that the artificial pressure becomes important outside of $\sim 3 h_{50}^{-1}$ $\mathrm{Mpc}$ and peaks at about $5 h_{50}^{-1} \mathrm{Mpc}$, consistent with the position of the shock front derived from the entropy profile in Figure 8f. Within an Abell radius, $R_{\mathrm{A}}=3 h_{50}^{-1} \mathrm{Mpc}$, the artificial pressure is negligible.

The total radial acceleration is negative within $2 h_{50}^{-1} \mathrm{Mpc}$ with an amplitude of roughly $15 \%$ that of the pressure gradient. The velocity amplitude associated with this acceleration, $\sim 500 \mathrm{~km} \mathrm{~s}^{-1}$, is inconsistent with the lack of radial inflow seen in the gas velocity field. The solution to this apparent inconsistency is in noting that the calculated pressure gradient (line 2) contains only a thermal contribution, with no allowance for kinetic pressure due to the residual velocity dispersion of the gas. The ratio of kinetic to thermal pressures is $\beta_{\text {gas }}=$ $\sigma_{\mathrm{gas}}^{2} /\left(k T / \mu m_{p}\right)$, the measured value of which is $\beta_{\mathrm{gas}}=0.13$. Thus, inclusion of kinetic pressure offsets the net negative acceleration in line 4 and makes the total radial acceleration consistent with the hydrostatic equilibrium implied by the velocity field.

The second question to address is the accuracy of the mass modeling assumed in the isothermal $\beta$-model. Figure 11 shows radial profiles of both the gas and the binding (i.e., gas + DM) mass distributions at $z=0$. The profiles are computed in radial bins containing 50 particles and are truncated at a radius where the mean interior density $\delta \rho / \rho=170$ in an attempt to encompass only the relaxed portion of the cluster (Kaiser 1986). This overdensity is reached at a radius $r \simeq 3.5 h_{50}^{-1} \mathrm{Mpc}$, in good agreement with the "edge" of the cluster one would determine from the acceleration data in Figure 10 or the velocity data in Figures 8 and 9. These data are fitted to the isother$\operatorname{mal} \beta$ form

$$
\rho_{s}(r)=\rho_{0, s}\left[1+\left(r / r_{c, s}\right)^{2}\right]^{-3 \beta_{s} / 2},
$$

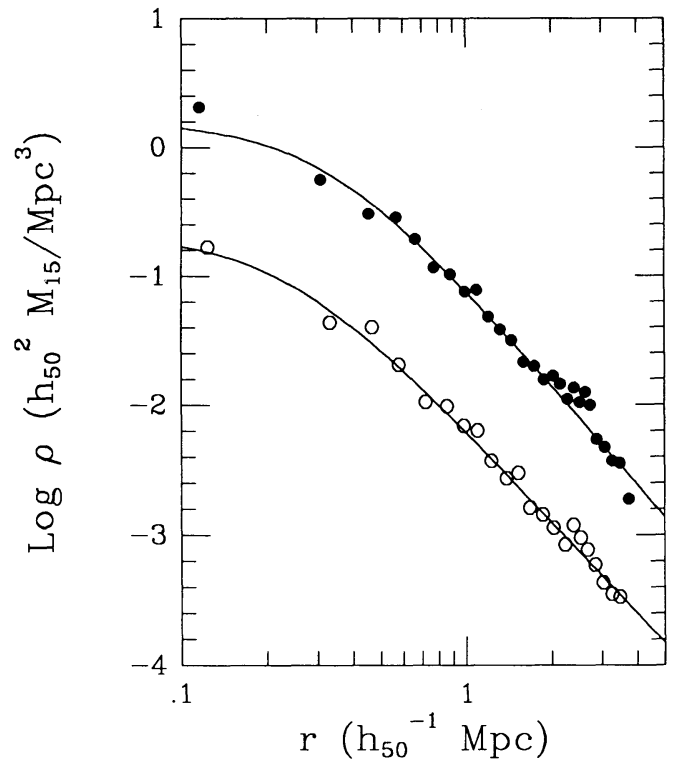

Fig. 11.-Binding mass ( filled circles) and gas mass (open circles) profiles at $z=0$ along with fits to the isothermal $\beta$-model profile, eq. (25). Parameters of the fits are given in Table 3. 
TABLE 3

Mass Profile Fits

\begin{tabular}{cccccc}
\hline \hline Mass & $\left(10^{15} h_{50}^{2}{\stackrel{\rho}{M_{\odot}}}_{\odot} \mathrm{Mpc}^{-3}\right)$ & $\left(h_{50}^{-1} r_{\mathrm{kpc}}\right)$ & $\beta$ & $\bar{\chi}^{2}$ & $N_{\mathrm{df}}$ \\
\hline Binding $\ldots \ldots$ & $1.6 \pm 0.5$ & $320 \pm 50$ & $0.85 \pm 0.04$ & 2.2 & 25 \\
Gas $\ldots \ldots \ldots \ldots$ & $0.21 \pm 0.04$ & $230 \pm 40$ & $0.77 \pm 0.03$ & 1.3 & 20
\end{tabular}

where the subscript $s$ denotes either gas $(g)$ or binding mass $(b)$. Values of the free parameters in the fit are shown in Table 3. Uncertainties of $14 \%$ in the density of each bin are based on the assumption of Poisson noise in the particle count. The reduced $\chi^{2}$ and number of degrees of freedom are given in Table 3. The poorer fit to the binding mass arises mainly from a lump of material between 2 and $3 \mathrm{Mpc}$. This lump is a remnant of the merger event which occurred at $z \sim 0.2$; the material is near the apastron of its first orbit and will turn around and fall back into the cluster center on a time scale $\sim 10^{9}$ yr. In a few crossing times, the material will phase-mix and blend into the general cluster distribution.

The fitted core radii are not much larger than the experimental resolution limits, the binding mass core radius is $r_{c, b}=320$ $\pm 40 h_{50}^{-1} \mathrm{kpc}$ and that of the gas is $r_{c, g}=230 h_{50}^{-1} \mathrm{kpc}$. The quoted uncertainty in the former value would suggest it is $4 \sigma$ different from the gravitational softening $\epsilon=150 h_{50}^{-1} \mathrm{kpc}$. However, the magnitude of the softened acceleration is $50 \%$ lower than an $r^{-2}$ law at a separation $r=2 \epsilon$, so the effect of softening is still considerable at radii $\sim 300 h_{50}^{-1} \mathrm{kpc}$. Also, results from a higher resolution simulation presented in $\S \mathrm{V}$ confirm that this core radius is an artifact of the gravitational softening and so cannot be claimed to be resolved. The core radius of the gas distribution is comparable to the resolution limit of the SPH and so is also unresolved. These results suggest the possibility that traditional "cores" of constant density are not a universal phenomenon in clusters, an idea supported by some optical studies of galaxy density profiles (Beers and Tonry 1986; Merrifield and Kent 1989).

The outer profile slopes are known to better accuracy, since they are determined by a much larger amount of mass than are the core radius and central density. The values of $\beta_{b}=0.85$ \pm 0.03 and $\beta_{g}=0.77 \pm 0.02$ indicate that the dark matter profile is slightly steeper than that of the gas, although this statement is sensitive to the choice of cut off radius for the fit. The King model of equation (23), which assumes $\beta_{b}=1$, does not provide the best description for the form of the binding potential. The binding mass density in the outer parts of the cluster falls off as $\sim r^{-2.5}$ rather than $r^{-3}$. A similar radial density dependence was found by West, Dekel, and Oemler (1987) for clusters simulated in a hierarchial model with spectral index $n=-1$, roughly that of CDM on the mass scale of rich clusters.

The gas density outer profile $\beta_{g}=0.77 \pm 0.02$ is consistent with the value obtained by fitting the surface brightness profile $\beta_{\mathrm{fit}}=0.76 \pm 0.02$ using an isothermal temperature assumption. This indicates that reliable estimates of gas mass may be made observationally under the isothermal assumption if the gas temperature $T$ is known. Does the value of $\beta_{\mathrm{fit}}$ also correctly reflect the ratio of specific energies $\sigma^{2} /\left(k T / \mu m_{p}\right)$ ? At $z=0$, the dark matter velocity dispersion $\sigma=1150 \mathrm{~km} \mathrm{~s}^{-1}$ and mean gas temperature $T=7.2 \mathrm{keV}$ measured within an Abell radius provide a true value of $\beta=1.2$. The "spectroscopic" estimate $\beta_{\text {spec }}$ will reflect this value if measurement errors are small.
Thus, the simulated cluster displays the same $\beta$-discrepancy as observed clusters.

How this discrepancy arises can be understood by writing the equation of hydrostatic equilibrium for an isothermal gas including the effect of kinetic pressure which yields

$$
3 \frac{k T}{\mu m_{p}} \beta_{\mathrm{fit}}\left(1+\beta_{\mathrm{gas}}\right)=\frac{G M_{b}(r)}{r}
$$

and the corresponding Jeans equation for the dark matter including the effects of orbital anisotropy

$$
3 \sigma_{r}^{2}\left[\beta_{\mathrm{DM}}-\frac{2}{3 A(r)}\right]=\frac{G M_{b}(r)}{r} .
$$

Taking the ratio of these two yields

$$
\frac{\sigma^{2}}{k T /\left(\mu m_{p}\right)} \equiv \beta=\frac{\beta_{\mathrm{fit}}\left(1+\beta_{\mathrm{gas}}\right)}{\beta_{\mathrm{DM}}-2 / 3 A(r)} \text {. }
$$

Plugging in appropriate values $\beta_{\mathrm{fit}} \sim \beta_{g} \simeq 0.76, \beta_{\mathrm{gas}}=0.13$, $\beta_{\mathrm{DM}}=0.85$ and $A \simeq 0.2$ produces a value $\beta=1.2$, which is the true value. The fiducial form of the hydrostatic isothermal $\beta$-model assumes $\beta_{\text {gas }}=0, \beta_{\mathrm{DM}}=1$, and $A(r)=0$, which leads to $\beta=\beta_{\mathrm{fit}}$. The source of the $\beta$-discrepancy is thus attributable to ignoring incomplete thermalization of the gas and poorly modeling the dark matter density distribution by assuming $\beta_{\mathrm{DM}}=1$ and $A(r)=0$. Each of these corrections is only a $\sim 15 \%$ effect, but they all push in the same direction of making $\beta>\beta_{\mathrm{fit}}$.

Finally, we can ask the simple question of how accurate are the binding masses estimated using the simplest form of the hydrostatic isothermal $\beta$-model. The predicted form of the binding mass using equations (1) and (24) is

$$
\begin{aligned}
M_{b}(r) & =3 \beta_{g} G^{-1} \frac{k T}{\mu m_{p}} r \frac{\left(r / r_{c, g}\right)^{2}}{1+\left(r / r_{c, g}\right)^{2}} \\
& =1.1 \times 10^{14} \beta_{g} \frac{T}{\mathrm{keV}} \frac{r}{\mathrm{Mpc}} \frac{\left(r / r_{c, g}\right)^{2}}{1+\left(r / r_{c, g}\right)^{2}} h_{50}^{-1} M_{\odot} .
\end{aligned}
$$

Figure 12 shows the comparison of the true mass profile with this estimate based on the fitted gas parameters $\beta_{g}$ and $r_{c, g}$ in Table 3 and temperature $T=7.2 \mathrm{keV}$. The model underestimates the true binding mass by roughly $30 \%$ at radii smaller

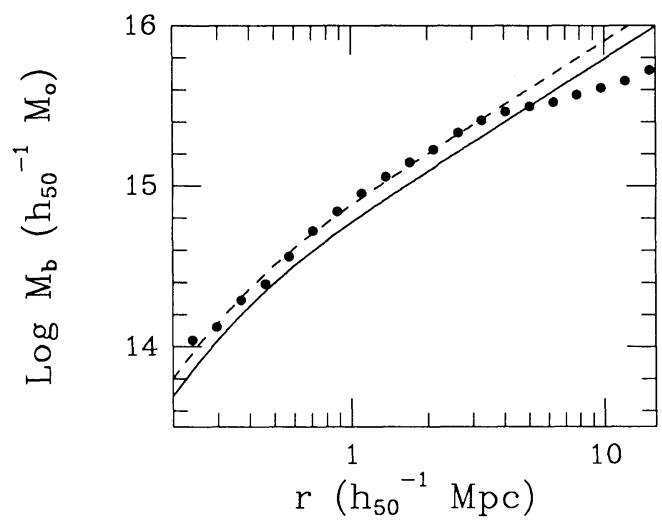

FIG. 12.-Binding mass predictions based on the hydrostatic, isothermal $\beta$-model compared to the actual enclosed mass profile (filled circles) of the model cluster at $z=0$. The solid line is the uncorrected estimate given by eq. (29), while the dashed line shows this estimate corrected by a factor of 1.3 . 
than the Abell radius. Roughly half of this underestimate is due to ignoring gas kinetic motions. The remaining $\sim 15 \%$ effect may arise from the fact that the temperature is not exactly isothermal. Using the local values of the temperature in the above estimate would boost the estimated mass around $1 h_{50}^{-1}$ Mpc by about $15 \%$. At any rate, taking these effects into account by simply multiplying equation (29) by a fudge factor of 1.3 yields an excellent fit to the mass profile within an Abell radius, as indicated by the dashed line in Figure 12.

\section{COMPARISON WITH COMA AND A2256}

It is instructive to make a more detailed comparison of the model with Coma and A2256 - two rich, nearby clusters studied extensively in X-rays by Hughes (1989) and Fabricant, Kent, and Kurtz (1989), respectively. It should be emphasized that a carbon copy of a particular cluster is impossible to guarantee from Gaussian random initial conditions. Some level of disagreement between the model and either Coma or A2256 is therefore to be expected and need not be considered a failure on the part of the model. To properly address the general compatability of the models with observations requires examination of ensemble properties (parameter correlations, abundance distributions, etc.) which will be done in Paper II. However, it is worth examining the similarities and differences between the model cluster and Coma and A2256 in order to gain some insight into the dynamical history of these real clusters. Table 4 presents a summary of observed quantities for the observed clusters along with those for the model when viewed at the appropriate distance.

With a rest frame $2-10 \mathrm{keV}$ luminosity of $L_{x}=7.5 \times 10^{44}$ ergs $\mathrm{s}^{-1}$, the model is as luminous as Coma (Lahav et al. 1989) and slightly dimmer than A2256 with $L_{x}=1.0 \times 10^{45} \mathrm{ergs} \mathrm{s}^{-1}$ (Fabricant, Kent, and Kurtz 1989). Comparison of the surface brightness profiles shown in Figure 13 indicates that the emission spanning roughly two decades in flux from $10^{-2}$ to $10^{-4}$ counts $\mathrm{s}^{-1}$ arcmin $^{-2}$ is very similar in the model and observed clusters. Near the center, the model tends to produce more $\mathrm{X}$-rays than the real clusters. The central excess seen in a simulation without radiative cooling suggests that processes which tend to lower the central gas density may be important in non-centrally concentrated clusters (the $\mathrm{nXD}$ classification of Jones and Forman 1984) such as Coma and A2256. Thermal feedback during the galaxy formation era to preheat the gas to $\sim 10^{7} \mathrm{~K}$ (discussed in the next section), heating by galaxy motions and conduction (assuming a temperature inversion as

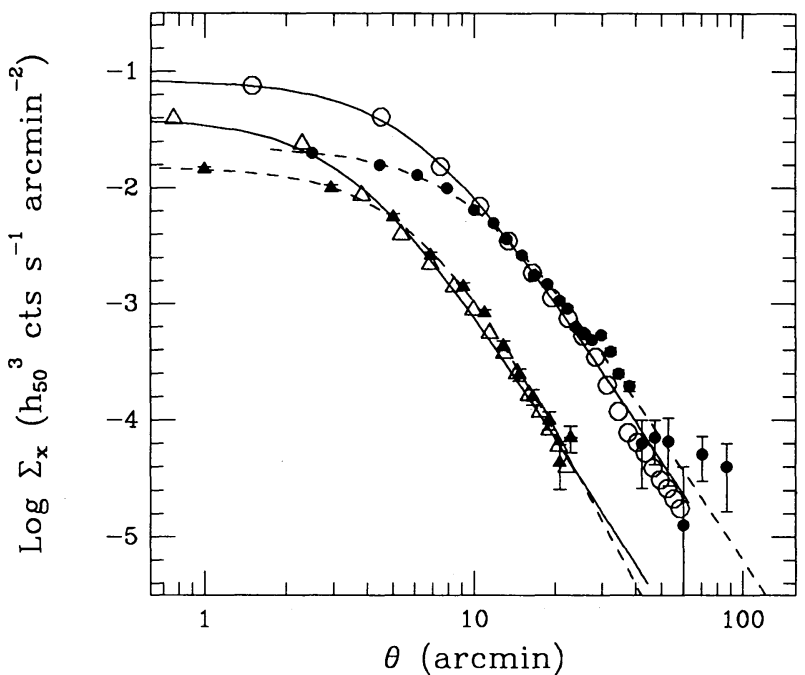

Fig. 13.-Comparison of the model cluster's surface brightness profile with data for Coma (filled circles) and A2256 (filled triangles). The model distribution at $z=0$ is placed at a redshift $z=0.023$ to compare with Coma, with results shown by open circles. The model output at $z=0.07$ is adjusted to the distance of A2256 $(z=0.06)$ to produce the open triangles. Parameters from fits to these data are given in Table 4.

seen in the model) are all possible mechanisms. Note, however, that the nXD class is actually a minority of all clusters, only eight out of the 46 clusters in the sample of Jones and Forman belong to this class. The majority of clusters have smaller core radii and higher central surface brightness, comparable to that seen in the model.

The mean, mass-weighted temperature of the gas in the simulation $T=7.2 \mathrm{keV}$ is comparable to the mean temperature of the ICM in Coma $T=7.5 \pm 0.2 \mathrm{keV}$ (Hughes 1989) and A2256 $T=7.4 \pm 1.0 \mathrm{keV}$ (Fabricant, Kent, and Kurtz 1989). The radial distribution of gas temperature is not known for A2256, but there are some indications for Coma that the gas is close to, but not perfectly, isothermal. Hughes, Gorenstein, and Fabricant (1988) fit EXOSAT ME spectra taken in four $45^{\prime}$ fields, one centered on Coma and the other three displaced $45^{\prime}$ (roughly $2 h_{50}^{-1} \mathrm{Mpc}$ ) to the south, east, and west, respectively. They found the center field to be $\sim 15 \%$ hotter than the off-center fields, indicating a temperature profile falling outside of $\sim 1 h_{50}^{-1} \mathrm{Mpc}$. Hughes et al. (1988)

TABLE 4

Comparison with CoMa AND A2256

\begin{tabular}{|c|c|c|c|}
\hline Observation & Model & Coma $^{a}$ & $A 2256^{b}$ \\
\hline 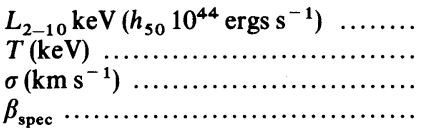 & $\begin{array}{c}7.5 \\
7.2 \\
1150 \\
1.2\end{array}$ & $\begin{aligned} 7.4 & \pm 0.4 \\
7.5 & \pm 0.2 \\
1130 \pm 220^{c} & \pm 0.4\end{aligned}$ & $\begin{array}{c}10 \pm 1 \\
7.4 \pm 1.0 \\
1300 \pm 100 \\
1.4 \pm 0.3\end{array}$ \\
\hline $\begin{array}{l}\beta_{\text {fit }} \ldots \ldots \ldots \ldots \ldots \ldots \ldots \ldots \ldots \ldots \ldots \ldots \\
\Sigma_{0}\left(10^{-3} \text { IPC counts s }{ }^{-1}\right) \ldots \ldots \ldots \ldots \ldots\end{array}$ & $\begin{array}{l}0.76 \pm 0.02 \\
84 \pm 5 \\
40 \pm 13 \\
240 \pm 8\end{array}$ & $\begin{array}{c}0.75 \pm 0.02 \\
23 \pm 2 \\
\cdots \\
380 \pm 15\end{array}$ & $\begin{array}{c}0.83 \pm 0.06 \\
\cdots \\
15 \pm 5 \\
520 \pm 50\end{array}$ \\
\hline $\begin{array}{l}\text { Predicted } M_{b}\left(<R_{A}\right)\left(10^{15} M_{\odot}\right) \ldots \ldots \\
\text { Actual } M_{b}\left(<R_{A}\right)\left(10^{15} M_{\odot}\right) \ldots \ldots \ldots\end{array}$ & $\begin{array}{l}2.4 \\
2.3\end{array}$ & $\begin{array}{c}2.4 \\
?\end{array}$ & $\begin{array}{c}2.6 \\
?\end{array}$ \\
\hline
\end{tabular}

a-ray data taken from Hughes 1989

b X-ray and optical data taken from Fabricant et al. 1989.

c From Fitchett and Webster 1987. 
performed spectral fits to data from the Tenma satellite subject to constraints provided by images of Coma from Einstein. Their analysis indicated a departure from isothermality at the $90 \%$ confidence level. To properly compare these results with expectations from the numerical model would require simulated observations and data reductions performed on the model in a manner as similar as possible to the actual observations. This is clearly worth doing but is beyond the scope of the present paper. The radial temperature distribution of the gas shown in Figure $8 b$ indicates that the temperature does fall by $\sim 20 \%$ between 1 and $2 h_{50}^{-1} \mathrm{Mpc}$, roughly the amount seen in Coma. However, one should be wary of such a simple eyeball estimate when looking for such a small effect as it is possible that the large emission measure from the cooler gas interior to $1 h_{50}^{-1} \mathrm{Mpc}$ will produce a lower central temperature estimate.

The velocity dispersion $\sigma=1150 \mathrm{~km} \mathrm{~s}^{-1}$ of the model represents the rms one-dimensional velocity of particles within an Abell radius of the cluster center. There is some ambiguity in the value of $\sigma$ for Coma. The listed value of $\sigma=1130 \pm 220$ $\mathrm{km} \mathrm{s}^{-1}$ is taken from an analysis by Fitchett and Webster (1987) of the Kent and Gunn (1982) data set. Fitchett and Webster applied a standard " $3 \sigma$ clipping" algorithm (Yahil and Vidal 1977) to galaxies in the central $20^{\prime}$ of Coma, resulting in 54 candidate cluster members which produced the quoted velocity dispersion and uncertainty. Their statistical analysis also indicated the possible presence of two subcomponents within the cluster with individual velocity dispersions of $1240 \pm 230 \mathrm{~km} \mathrm{~s}^{-1}$ and $880 \pm 140 \mathrm{~km} \mathrm{~s}^{-1}$. The situation is further complicated by the fact that the measured velocity dispersion is a function of projected radius from the cluster center. However, this is also true for the simulation, as shown in Figure 14. Qualitatively the model profile resembles that of Coma-rising in the center to a maximum at roughly $10^{\prime}$ and falling at larger radii. All clusters exhibit the $\beta$-discrepancy at some level. Values of $\beta_{\mathrm{fit}}=0.76 \pm 0.02, \quad 0.75 \pm 0.02$, $0.83 \pm 0.02$ are determined from the surface brightness fits of the model, Coma and A2256, respectively while the spectroscopic values are $\beta_{\text {spec }}=1.2,1.1 \pm 0.4,1.4 \pm 0.3$. The large uncertainty in the value of $\beta_{\text {spec }}$ for Coma reflects its controver-

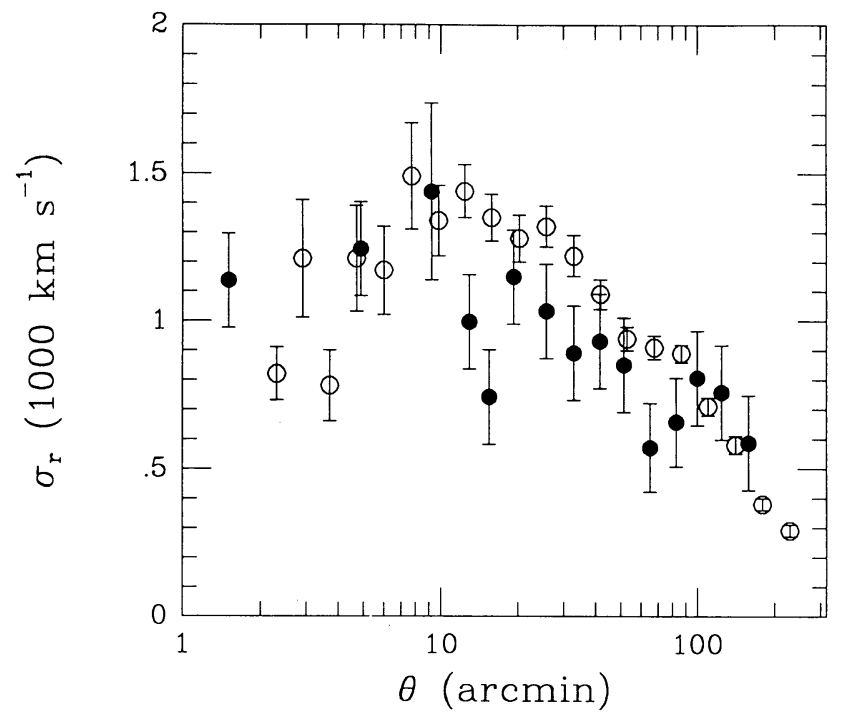

FIG. 14.- - Line-of-sight velocity dispersion $\sigma_{r}$ as a function of projected distance from the cluster center $\theta$ is shown for Coma (filled circles) and the model viewed along the $z$-axis of the simulation (open circles).

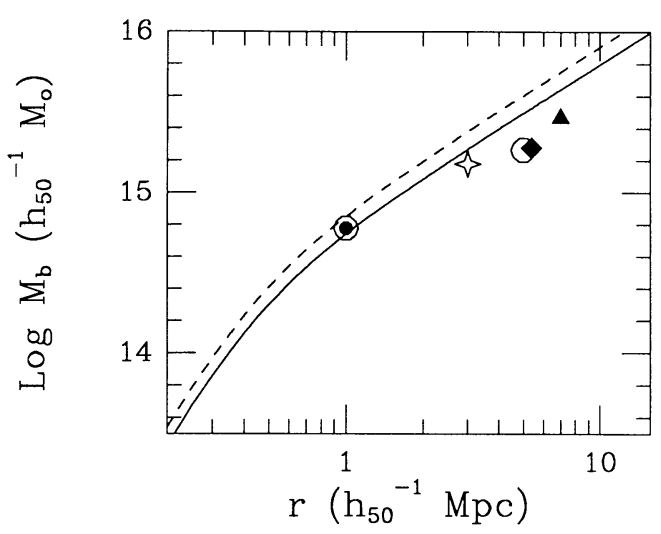

FIG. 15.-Binding mass estimates for the Coma cluster based on the hydrostatic, isothermal $\beta$-model without (solid) and with (dashed) the correction factor of 1.3. Data points refer to previous estimates in the literature based on optical (Kent and Gunn 1982, solid triangle; The and White 1986, solid diamond; and Merritt 1987, solid circle) and X-ray data (Hughes et al. 1988, open star, and Hughes 1989, open circles).

sial nature- one could have picked a central temperature of $9.5 \mathrm{keV}$ (Hughes 1989) and combined it with a lower estimate of $\sigma$ at larger radii (Fig. 14) to produce a value $\beta_{\text {spec }}<1$.

The similarities between the model and the observed clusters prompt an estimate of the binding mass of Coma and A2256 to be made using the corrected hydrostatic isothermal $\beta$-model. Figure 15 shows the predicted binding mass profile for Coma based on equation (29) with the observed values of $T, \beta_{\mathrm{fit}}$, and $r_{c}$ both with (dashed line) and without (solid line) the correction factor of 1.3. Also shown are previous estimates based on optical (Kent and Gunn 1982; The and White 1986; Merritt 1987) and X-ray (Hughes 1989) data. The value of the binding mass at $1 h_{50}^{-1} \mathrm{Mpc}$ appears well determined at $M=7.0$ $\times 10^{14} h_{50}^{-1} M_{\odot}$ to an accuracy of around $10 \%$. The estimates at large radii are sensitive to the run of density in the assumed mass model. For the optical studies with a mass-traces-light assumption, the mass at large radii increases weakly with radius $M(r) \propto r$. This generates the discrepancy in the predictions at $r \gtrsim 5 h_{50}^{-1} \mathrm{Mpc}$. However, the dynamics of the model indicates that the virial regions of Coma and A2256 do not extend beyond an Abell radius, so mass estimates based on the virial theorem cannot be trusted beyond radii $r \sim 3 h_{50}^{-1} \mathrm{Mpc}$ (as seen in Fig. 12). Estimates for the binding mass within a Abell radius for Coma and A2256 are listed in Table 4, including the factor of 1.3. The uncorrected estimate for Coma is $M_{b} \simeq 1.8 \times 10^{15} M_{\odot}$, close to the value of $M_{b} \simeq 1.5 \times 10^{15}$ $M_{\odot}$ produced by Hughes et al. (1988).

\section{SENSITIVITY TO INITIAL CONDITIONS AND NUMERICAL PARAMETERS}

In this section, results of six runs designed to test parameter sensitivity are presented and discussed. Table 5 lists the models and describes their distinguishing characteristic relative to the model discussed in the previous sections, referred to as model M. Model DT uses a time step which is twice the value used in model M. Models V1 and V2 vary the artificial viscosity coefficients, spanning a factor $\sim 2$ range around the values used in model $M$. The effect of the initial gas temperature is explored in runs $\mathrm{T} 1$ and $\mathrm{T} 2$, for which $T_{i}=10^{7}$ and $10^{8} \mathrm{~K}$, respectively. Finally, model CP is a high-resolution run with 65,536 total particles which includes radiative losses in the gas thermal 
TABLE 5

\begin{tabular}{|c|c|}
\hline \multicolumn{2}{|c|}{ DeSCRIPTION OF TEST Runs } \\
\hline Run & Description \\
\hline $\mathbf{M}$ & "Standard" model \\
\hline DT & $\delta t=3.8 \times 10^{7} h_{50}^{-1} \mathrm{yr}$ \\
\hline V1 & $\alpha_{1}=1, \alpha_{2}=3.5$ \\
\hline V2 & $\alpha_{1}=0.25, \alpha_{2}=1.75$ \\
\hline T1 & $T_{i}=10^{7} \mathrm{~K}$ \\
\hline T2 & $T_{i}=10^{8} \mathrm{~K}$ \\
\hline ............ & Cooling on, $N_{p}=65,536$ \\
\hline
\end{tabular}

history. The initial particle displacement field for the $32^{3}$ run was determined from that of the $16^{3}$ model by interpolation using a Gaussian kernel with $\sigma=0.85(L / 16)$. In all other runs, the initial displacement field is identical to that of model $\mathrm{M}$.

The final density and temperature profiles for these runs are shown in Figure 16. The limiting hydrodynamic resolution $h_{\min }$ of model $\mathrm{M}$ is indicated for comparison. Decreasing the time step a factor 2 has the effect of degrading the central resolution because the Courant condition presents a more stringent limit on the value of $h$ than does the discreteness condition in the cluster center. Thus, model DT departs from the model M solution for radii $r \lesssim 300 h_{50}^{-1} \mathrm{kpc}$. The enhanced density and cooler temperatures seen in this central region are numerical artifacts arising from the inability to resolve pressure gradients on this scale. Kinetic pressure is responsible for roughly half of the support of the core. The solutions outside $300 h_{50}^{-1} \mathrm{kpc}$ are in very good agreement. Varying the viscosity coefficients has a smaller effect on the solutions. For radii $r \gtrsim 300 h_{50}^{-1} \mathrm{kpc}$, there is good consistency among the solutions.

Since gas will not be compressed back into the cluster if put on a sufficiently high adiabat, varying the initial temperature has no significant effect until temperatures at which the initial entropy approaches the minimum value resolved in the cluster center. This will be true for redshifts $z \lesssim 10$ where Compton cooling is inefficient. The ambient cosmological density at the initial redshift $z_{i}=7$ is 512 times larger than the present cosmological density. Thus to affect the cluster mass presently within a radius where the overdensity $\delta \rho / \rho=512$ for a cluster with plasma temperature $T_{c}$ would require an initial temperature $T_{i} \geq T_{c} \sim 10^{8} \mathrm{~K}$. Model T1, with $T_{i}=10^{7} \mathrm{~K}$, should limit the central density to $\delta \rho / \rho \lesssim 1.6 \times 10^{4}$ or $n \lesssim 10^{2.1}$ $\mathrm{cm}^{-3}$. This is within a factor of 2 of the value seen in Figure 16. If the initial temperature is raised to $10^{8} \mathrm{~K}$ (model T2), then gas with densities $n \gtrsim 10^{-3.6} h_{50}^{-2} \mathrm{~cm}^{-3}$ will be on too high an adiabat to collapse. Again, the simulation shows that the final cluster central density is limited to within a factor of 2 of this value. The energies involved in these hot initial conditions are not too restrictive. The internal energy of isothermal gas is

$$
U=4 \times 10^{61}\left(\frac{M_{\text {gas }}}{10^{13} h_{50}^{-1} M_{\odot}}\right)\left(\frac{T}{10^{7} K}\right) \text { ergs . }
$$

To produce this much energy from $N_{s}$ sources each with $M_{s}=$ $10^{10} h_{50}^{-1} M_{\odot}$ of baryonic fuel would require an efficency

$$
\epsilon=\frac{U}{N_{s} M_{s} c^{2}}=\frac{0.002}{N_{s}}\left(\frac{M_{\mathrm{gas}}}{10^{13} h_{50}^{-1} M_{\odot}}\right)\left(\frac{T}{10^{7} \mathrm{~K}}\right) .
$$

Thus, $10^{13} h_{50}^{-1} M_{\odot}$ of gas in the core of a rich cluster could be preheated to a temperature up to $10^{7} \mathrm{~K}$ by a single source with a rather modest efficiency $\epsilon \sim 10^{-3}$ characteristic of that expected from normal stellar processes alone (Bookbinder et al. 1980).

The higher resolution run with radiative cooling exhibits a poorly resolved cooling flow which consumes $\sim 10^{13} h_{50}^{-1} M_{\odot}$ of baryons within $100 h_{50}^{-1} \mathrm{kpc}$ of the cluster center by the present day. The density profile is therefore sharply peaked toward the center, with $\rho(r) \propto r^{-3.5}$ for radii between 100 and $200 h_{50}^{-1} \mathrm{kpc}$. The temperature plummets almost an order of magnitude in this region. Outside of $300 h_{50}^{-1} \mathrm{kpc}$, the density profiles of model CP follows closely that of model $\mathrm{M}$. The temperature between 300 and $700 h_{50}^{-1} \mathrm{kpc}$ is larger by up to $50 \%$ in the model CP. The source of this difference is not entirely clear: it may be due to better resolution of shocks in the early stages of the cluster formation. Much better agreement in the temperature profiles is found at larger radii. The mass weighted temperatures within an Abell radius for models $\mathrm{CP}$ and $\mathrm{M}$ agree to within $7 \%$.
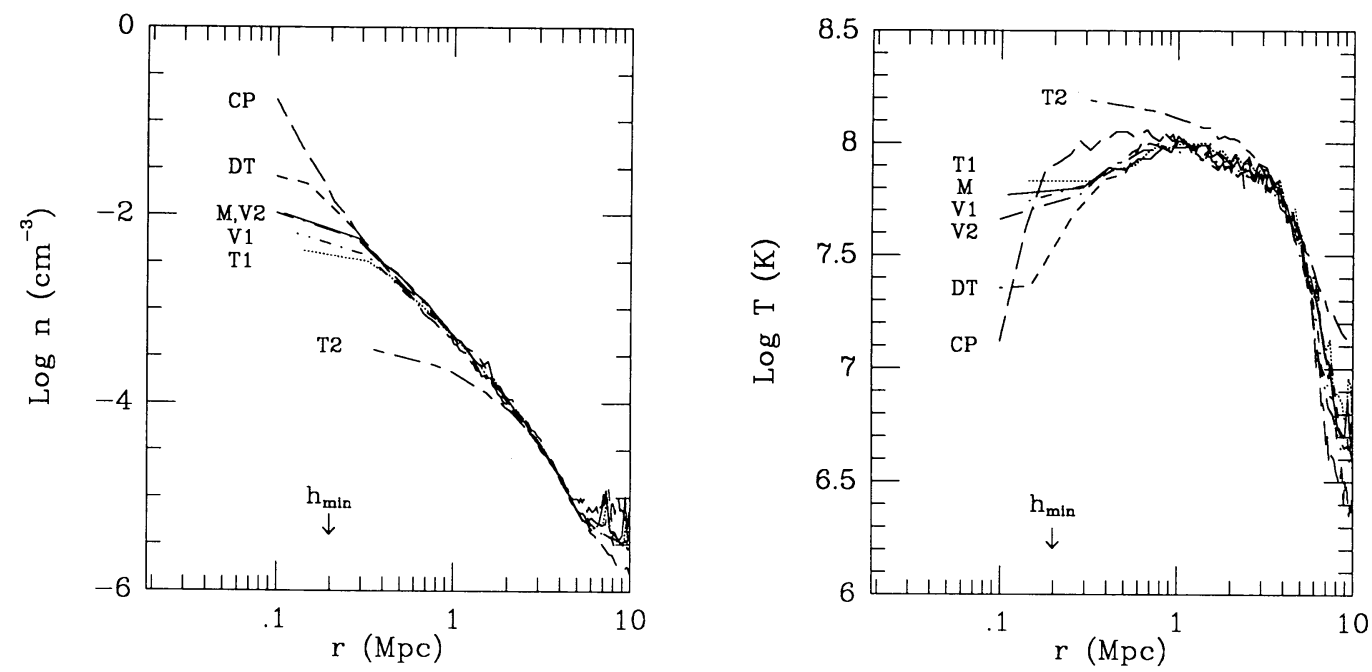

Fig. 16.-Density and temperature profiles for the test runs and the fiducial model $M$ at the final epoch. The resolution limit $h_{\min }$ for the $16^{3}$ particle models is indicated. 


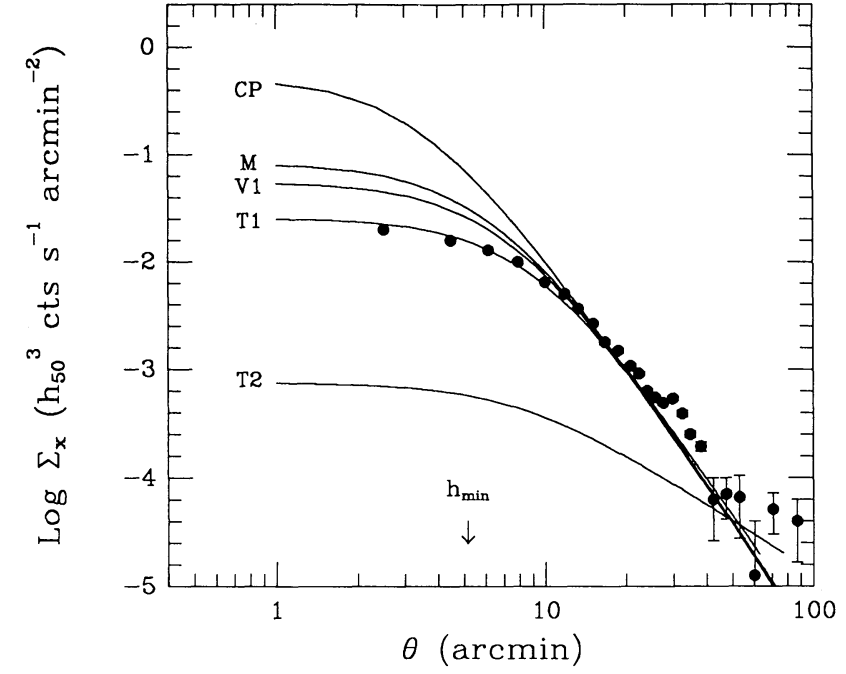

Fig. 17.-Fits to the X-ray surface brightness of selected models are compared to the data for Coma (solid circles). Model T1 which began with an enhanced ICM gas temperature $T_{i}=10^{7} \mathrm{~K}$ at $z_{i}=7$ provides a good match to the Coma central brightness.

Fits to profiles of the cluster X-ray emission resulting from some of these test runs are shown in Figure 17. The Coma Cluster data are shown for comparison. The cooling flow model CP exhibits significantly enhanced core emission, 20 times above the observed central level in Coma. The total 2-10 $\mathrm{keV}$ emission of this run is double that of model $\mathbf{M}$ (and Coma), with all of the extra emission coming from the inner $\sim 200 h_{50}^{-1} \mathrm{kpc}$. Model V1 with slightly larger viscosity coefficients has a central emissivity $\sim 25 \%$ lower than model $\mathrm{M}$, but outside the unresolved core agreement is good. Model V2 is indistinguishable from Model $\mathbf{M}$ on this plot and so is not shown.

Increasing the initial entropy to very high values, as in Model T2, produces very extended, low surface brightness emission. Since the collisionless component is not affected by the initial gas temperature, this cluster has a velocity dispersion roughly equal to that of Coma but an X-ray luminosity a factor 10 smaller. With the more modest initial temperature of $10^{7} \mathrm{~K}$ (Model T1), the central density and hence emissivity are reduced by small factors. In this case, the central surface brightness agrees very well with that observed in Coma.

To summarize, the tests show that the solution for the poorly resolved inner $\sim 300 h_{50}^{-1} \mathrm{kpc}$ region of the cluster is sensitive to numerical parameters and assumed physics. This region contains only $\sim 5 \%$ of the total mass within an Abell radius. The structure exterior to this core is much more robust. The central surface brightness and hence total luminosity can be varied according to the assumed input physics. The initial gas entropy can be used to limit the final central density of the cluster and thereby reduce its X-ray emission. Alternatively, gas on initially low adiabats can reach sufficiently high densities where radiative cooling becomes important. This serves to enhance the central gas density and X-ray surface brightness. Clearly, more work needs to done on resolving the important physical processes which compete to control the thermodynamics of the cluster core gas. Spherically symmetric models tailored to follow the evolution of the cluster core component (e.g., Meiksin (1989)) are presently more appropriate for addressing these issues in detail.
VI. SUMMARY

The thermodynamic history of the hot ICM in a cluster of Coma richness was examined using a numerical simulation incorporating three-dimensional hydrodynamics of a collisional baryonic component within a self-consistently evolving dark matter mass distribution. The model evolves to states at low redshifts whose X-ray and "optical" (i.e., collisionless) properties are strikingly similar to Coma and A2256. The elliptical X-ray isophotes seen at $z=0$ in the simulation are remnants of a merger event which took place at $z=0.2$, roughly $3 h_{50}^{-1}$ billion years ago. The similarity in morphology between the model's isophotes and those of Coma and A2256 hints strongly at the possibility that these clusters also suffered moderate merger events in the recent past. The model also indicates that clusters of Coma richness should display a SunyaevZel'dovich central decrement of $\sim 0.5 \mathrm{mK}$, a level comparable to that seen in A665 and A2218 (Uson and Wilkinson 1988; Birkinshaw 1989).

The central X-ray emission in the model is enhanced by a factor 2-3 relative to the real clusters, a direct result of the fact that the simulated density profiles of both the gas and dark matter show no signs of a resolved, constant density core. Larger simulations with resolving power to $\sim 100 h_{50}^{-1} \mathrm{kpc}$ also failed to generate a resolved core radius. Tests showed that the solution to the structure outside of $300 h_{50}^{-1} \mathrm{kpc}$, where $\sim 95 \%$ of the cluster mass resides, is robust.

The simulation exhibited the same discrepancy in the two ways of estimating the ratio of specific energies $\beta=$ $\sigma^{2} /\left(k T / \mu m_{p}\right)$ as is observed. An estimate based on the hydrostatic isothermal $\beta$-model and a fit to the surface brightness profile resulted in a value less than one while a direct measurement using the actual dark matter velocity dispersion $\sigma$ and gas temperature $T$ produced a value greater than one. The sources of the discrepancy were discovered to be incomplete thermalization of the gas $\sigma_{\text {gas }}^{2} \simeq 0.13\left(k T / \mu m_{p}\right)$ and poor modeling of the binding mass distribution by an isotropic King approximation to an isothermal sphere. However, it was demonstrated that estimates of the binding mass within an Abell radius of systems of Coma richness can be made to $\sim 10 \%$ accuracy with this model using a simple correction factor of 1.3 to account for the above omissions. When applied to Coma and A2256, this yields estimates for the binding mass within an Abell radius of 2.4 and $2.6 \times 10^{15} h_{50}^{-1} M_{\odot}$, respectively.

The simulated cluster was assumed to have formed in a CDM dominated universe with cosmological parameters $\Omega=1, \Omega_{\mathrm{ICM}}=0.1, h_{50}=1$, and a normalization expressed as a bias parameter of $b=1.7$. The gas density $\rho_{g}$, temperature $T$, mass $M_{g}$, luminosity $L_{x}$, central Sunyaev-Zel'dovich decrement $\Delta T$, and viewing redshift $z$ scale with baryon fraction $\Omega_{\mathrm{ICM}}$, Hubble constant $h_{50}$, and normalization $b$ according to

$$
\begin{aligned}
\rho_{g} & \propto h_{50}^{2} \propto \Omega_{\mathrm{ICM}} b^{-3}, \\
T & \propto b^{-1} \\
M_{g} & \propto h_{50}^{-1} \Omega_{\mathrm{ICM}}, \\
L_{x} & \propto h_{50} \Omega_{\mathrm{ICM}}^{2} b^{-3.5}, \\
\Delta T & \propto h_{50}^{2} \Omega_{\mathrm{ICM}} b^{-4}, \\
1+z & \propto b^{-1} .
\end{aligned}
$$

These scalings allow the results to be translated into different cosmological settings. The temperature is least sensitive to 
these parameters, depending only on the spectrum normalization. The density, luminosity, and SZ decrement are most sensitive to cosmology. Paper II will examine a catalog of simulated clusters and address the feasibility of using characteristics of the cluster population to place constraints on parameters of the CDM cosmology.
I am grateful to Ed Bertschinger for the generous use of his initial condition routine and to $M$. Davis and $C$. Lacey for helpful suggestions. This work was supported by The Miller Foundation of Basic Research in Science at the University of California, Berkeley and by the NSF through grant AST4517811.
Bahcall, J. N., and Sarazin, C. L. 1977, Ap. J. (Letters), 213, L99.

Bahcall, N. A., and Soniera, R. M. 1983, Ap. J., 270, 20.

Bardeen, J. M., Bond, J. R., Kaiser, N., and Szalay, A. S. 1986, Ap. J., 304, 15.

Beers, T. C., and Tonry, J. L. 1986, Ap. J., 300, 557.

Bertschinger, E. 1987, Ap. J. (Letters), 323, L103.

Bertschinger, E., and Juszkiewicz, R. 1988, Ap. J. (Letters), 334, L59.

Birkinshaw, M. 1989, in The Cosmic Microwave Background: 25 Years Later, ed. N. Mandolesi and N. Vittorio (Dordrecht: Kluwer), in press.

Blumenthal, G. R., Faber, S. M., Primack, J. R., and Rees, M. J. 1984, Nature, 311, 517.

Bond, J. R., and Efstathiou, G. 1984, Ap. J. (Letters), 285, L45.

Bookbinder, J., Cowie, L. L., Krolik, J. H., Ostriker, J. P., and Rees, M. 1980, Ap. J., 237, 647.

Cavaliere, A., and Fusco-Fermiano, R. 1976, Astr. Ap., 49, 137.

Cavaliere, A., Santangelo, P., Tarquini, G., and Vittorio, N. 1986, Ap. J., 305, 651 .

Cowie, L. L., Henriksen, M., and Mushotzky, R. 1987, Ap. J., 317, 593.

Cowie, L. L., and Perrenod, S. C. 1978, Ap. J., 219, 254

David, L. P., Forman, W., and Jones, C. 1990, Ap. J., 359, 29.

Davis, M., Efstathiou, G., Frenk, C. S., and White, S. D. M. 1985, Ap. J., 292, 371.

Edge, A. 1990, in Clusters of Galaxies, ed. W. R. Oegerle, M. S. Fitchett, and L. Danly (Cambridge: Cambridge University Press), in press.

Efstathiou, G., Davis, M., Frenk, C. S., and White, S. D. M. 1985, Ap. J. Suppl., $57,241$.

Efstathiou, G., and Eastwood, J. W. 1981, M.N.R.A.S., 194, 503.

Evrard, A.E. 1988, M.N.R.A.S., 235, 911. 1989, Ap. J. (Letters), 341, L71.

$1990 a$, in Clusters of Galaxies, ed. W. R. Oegerle, M. S. Fitchett, and L. Danly (Cambridge: Cambridge University Press), p. 287

- $1990 b$, in preparation (Paper II).

Fabian, A. C. 1988, in Hot Astrophysical Plasmas, ed. R. Pallavicini (Dordrecht: Kluwer), p. 251.

Fabricant, D. G., Kent, S. M., and Kurtz, M. J. 1989, Ap. J., 336, 77.

Fitchett, M., and Webster, R. 1987, Ap. J., 317, 653.

Frenk, C. S., White, S. D. M., Davis, M., and Efstathiou, G. 1988, Ap. J., 327, 507

Forman, W., and Jones, C. 1982, Ann. Rev. Astr. Ap., 20, 547.

Giacconi, R., et al. 1979, Ap. J.(Letters), 234, L1.

Gingold, R. A., and Monaghan, J. J. 1977, M.N.R.A.S., 181, 375.

Górski, K., Davis, M., Strauss, M. A., White, S. D. M., and Yahil, A. 1989, Ap. J., 344, 1 .

Gull, S. F., and Northover, K. J. E. 1975, M.N.R.A.S., 173, 585

Gunn, J. E., and Gott, J. R. 1972, Ap. J., 209, 1.

\section{REFERENCES}

Hockney, R. W., and Eastwood, J. W. 1981, Computer Simulation using Particles (New York: McGraw Hill).

Hughes, J. P. 1989, Ap. J., 337, 21.

Hughes, J. P., Gorenstein, P., and Fabricant, D. 1988, Ap. J., 329, 82.

Hughes, J. P., Yamashita, K., Okumura, Y., Tsumemi, H., and Matsuoka, M. 1988, Ap. J., 327, 615.

Johnson, M. W., Cruddace, R. G., Fritz, G., Shulman, S., and Friedman, H. 1979, Ap. J. (Letters), 231, L45.

Jones, C., and Forman, W. 1984, Ap. J., 276, 38

Kaiser, N. 1986, M.N.R.A.S., 222, 323.

Kaiser, N., and Lahav, O. 1989, M.N.R.A.S., in press.

Karzas, W., and Latter, R. 1961, Ap. J. Suppl., 6, 167.

Kellog, E. M., Baldwin, J. R., and Koch, D. 1975, Ap. J., 199, 299.

Kent, S. M., and Gunn, J. E. 1982, A.J., 87, 945.

Lahav, O., Edge, A. C., Fabian, A. C., and Putney, A. 1989, M.N.R.A.S., 238, 881.

Larson, R. B., and Dinerstein, H. L. 1975, Pub. A.S.P., 87, 911.

Lattanzio, J. C., Monaghan, J. J., Pongracic, H., and Schwarz, M. P. 1986, SIAM J. Sci. Stat. Comp., 7, 591.

Lea, S. M. 1976, Ap. J., 203569.

Meiksin, A. 1989, preprint.

Merrifield, M. R., and Kent, S. M. 1989, A.J., 98, 351.

Merritt, D. 1987, Ap. J., 313, 121.

Monaghan, J. J. 1985, Comp. Phys. Rept., 3, 71.

Mushotzky, R. 1984, Phys. Scripta, T7, 157 .

- 1988, in Hot Astrophysical Plasmas, ed. R. Pallavicini (Dordrecht: Kluwer), p. 114

Peebles, P. J. E. 1982, Ap. J., 257, 438

Perrenod, S. C. 1978, Ap. J., 226, 566.

Sarazin, C. L. 1986, Rev. Mod. Phys., 58, 1.

Spitzer, L. 1968, Diffuse Matter in Space (New York: Wiley).

Sunyaev, R. A., and Zel'dovich, Y. B. 1970, Ap. Space Sci., 6, 20

Takeda, H., Nulsen, P., and Fabian, A. 1984, M.N.R.A.S., 208, 461.

The, L. S., and White, S. D. M. 1986, A.J., 92, 1248.

Uson, J. M., and Wilkinson, D. T. 1988, in Galactic and Extragalactic Radio Astronomy, ed. G. L. Verschuur and K. I. Kellermann (New York: Springer Verlag), p. 603.

Weinberg, S. 1972, Gravitation and Cosmology (New York: Wiley).

West, M., Dekel, A., and Oemler, A. 1987, Ap. J., 316, 1.

White, R. E. 1990 , preprint.

White, S. D. M. 1976, M.N.R.A.S., 177, 717.

Yahil, A., and Ostriker, J. P. 1973, Ap. J., 185, 787

Yahil, A., and Vidal, N. V. 1977, Ap. J., 214, 347

Zel'dovich, Y. B., and Sunyaev, R. A. 1969, Ap. Space Sci., 4, 301.

AUgust E. EVRARD: Department of Astronomy, University of California, Berkeley, CA 94720 